\title{
A Novel Distributed Denial-of-Service Detection Algorithm
}

\author{
A Theris \\ Prevented to \\ the Faculiy of \\ California Polyfechnic State Unisersity. San Luis Obispo
}

\author{
In Pantial Fulfillment \\ of the Requirements for the Degree \\ Maver of Science in Computer Science
}

by

Brett Tsudama

June 2004 
AUTHORIZATION FOR REPRODUCTION OF MASTERS THESIS

I grant permission for the reproduction of this thesis in its entirety or any of its parts, without further authorization from me.
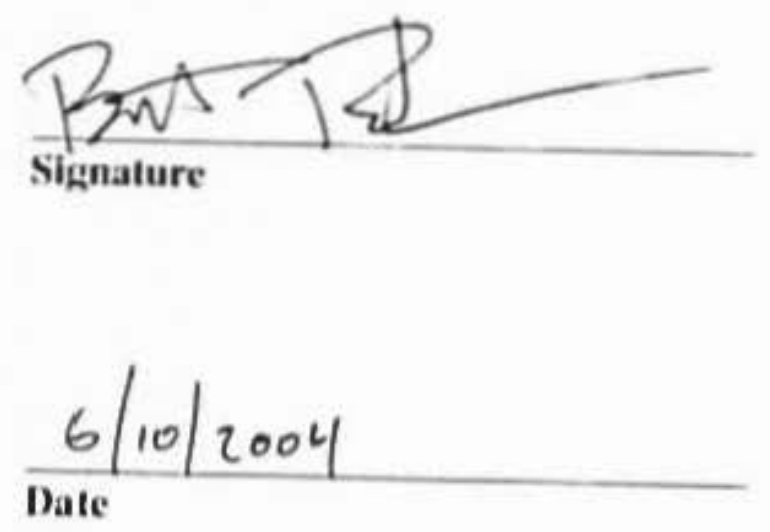


\section{APPROVAL PAGE}

TITLE: A Novel Distributed Denial-of-Service Detection Algorithm

AUTIIOR: Bret Tsudama

DATE SUBMITTED: June 10, 2004

Dr. Ilugh Smith

Advisor

Dr. Diama Keen

Committee Member

Dr. Mei.l Ling Liu

Committee Nlember
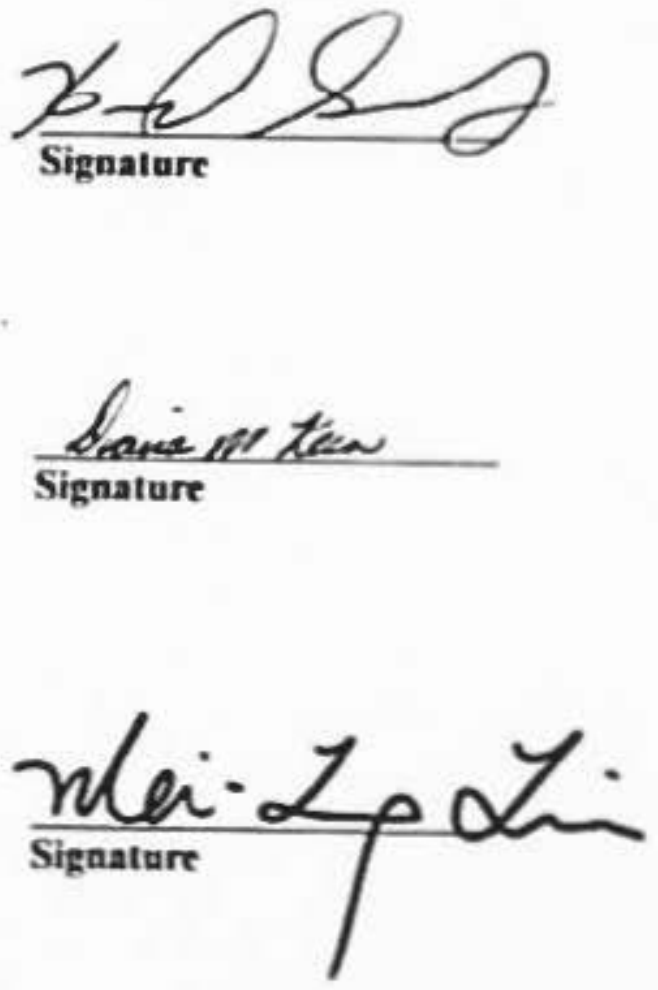


\section{ABSTRACT \\ A Novel Distributed Denlal-af-Service Detection Algorithm \\ Brett Tsudama}

Distributed Denial-of-Service (DDoS) attacks are becoming increasingly prevalent in the Internet today. A DDoS altack altempts to prevent a victim from providing a certain level of service to users, and the effects of a successful altack can range from minor inconveniences to major financial consecquences. This thesis presents a novel approach for the detection of a DDoS attack called Ratio-based SYN Flood Detection (RSD). RSD is an adaptive threshold algorithm and will dynamically adjust its belavior to achicve optimal performance in the face of varying traffic conditions. A methodology is presented which optimizes RSD to make it more generally suited for widespread deployment, and its efficacy is validaed through trace-driven simulations.

Network processors have been developed for routers in order to provide hardware-level performanee with the flexibility of a programmable architecture. Network processons are located on the edge roulers of a stub network, which is also where RSD is deployed. The applieability of implementing RSD on a network processor is explored, and initial results from this investigation are presented. 


\section{TABLLE OF CONTENTS}

I.IST OF TALLES....................................................................................

I.IST OF FIGURES........................................................................................

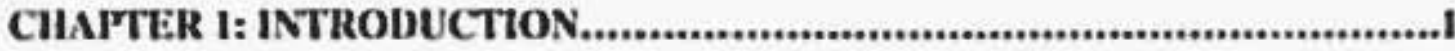

CHLATER 2: BACKGROUND AND REL_ATID WORKS................................

21 Types of DoS Altucks.....................................................................

2.2 DDaS Flooding Altacks....................................................................

2,1 Direct Allacks..............................................................4

2.2 .2 Renector Altucks..............................................................6

2.3 Solutions to the DDoS Problem.......................................................8

2.3.1 Altack Prevention and Preemptlon.....................................8

2.3.2 Attack Detection and Filtering..........................................9

2.3.2. I Spoofed IP Address Detection..................................11

2.3.2.2 Monitoring Trafle Characteristis.........................13

2.3.3 Altack Source Traceback and Identilieatlon.........................15

24 Netwark Pracessors.........................................................................15

25 Current Research on Network Processors.......................................17

CHAITER 3: RATIO-BASED SYN FI.OOD DETECTION ...........................19

3.1 SYN Floodling Attacks................................................................19

3.2 Ratio-laased SYN Flood Detectlon (RSD)......................................2I

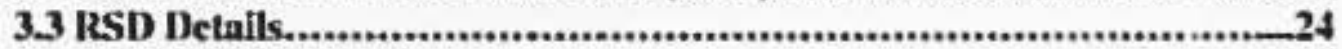

3.3.1 Varying a.........................................................................25

3.3 .2 Varying $\beta$.....................................................................26

3.3.3 Varying Atf..................................................................26

3.3.4 RSD Training Mlodule.......................................................26

3.3.5 Dynamically Adjusting At.............................................29

CHAITER 4: RSD IMIPLENIENTATION AND RESULTS...............................

4.1 DEC site results...........................................................................6.

4.2 L.BL, site results...........................................................................39

4.3 Discussion.....................................................................................40

4.3.1 SYN-dog Comparison......................................................40

CHAPTER 5: APPLICABILITY OF RSD TO NETWORK PROCESSORS.........43

5.1 Sinulator..................................................................................43

5.2 Implementation Results..........................................................4t

CILAPTER G: CONCLUSION......................................................................47

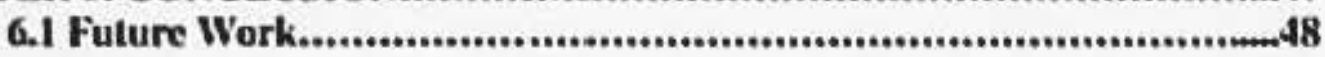

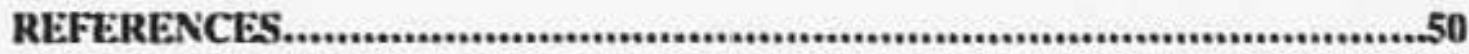




\section{LIST OF TAIJ.ES}

Table 1: Examples of common DDaS refertor attacks........................................

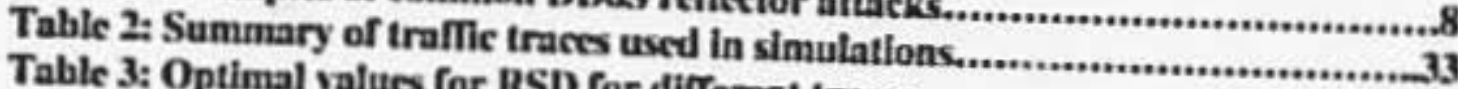

Table 3: Optimal values for RSD for different traces..........................................34 


\section{L.IST OF FIGURES}

Figure I: A typleal DDoS altack network.....................................................5

Figure 2: Flow of trafile in a direct attack and a reflector atuack.........................7

Figure 3: Possible locatlons for detection and riltering meclunism........................10

Figure 4: Network processor arehitectures....................................................17

Figure 5: TCP stales correspanding to normal connectlon establlshment and

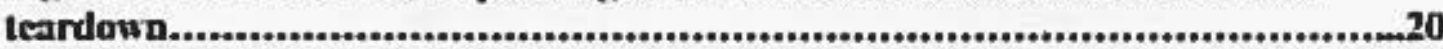

Figure 6: SYN and SYN-ACK packet counts at DIEC and I.[BI.........................23

Figure 7: EfTeets of varying $A t$ at DEC and L.BL......................................... 8

Figure 8: Average number of SYN-ACK packets olserved in each $A t$ for DI:C

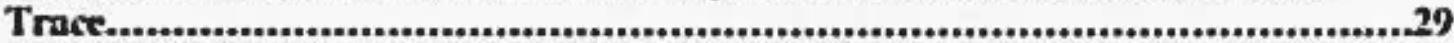

Figure 9: EJeets of varying $A t$ on DEC-I and DEC-2....................................31

Figure 10: Average number of SYN-ACK packets olsserved in ench di for DEC-1 and DEC-2..................................................................................31

Flgure 1I: Results from RSD training phase on DEC and L.BL traces...................34

Figure 12: Results from RSD simulatlons (DEC site)....................................36

Figure 13: Traltic traces with and without attack tralfic for DEC-PKT-I,

DEC-PKT-2, and DEC-PKT-3..................................................................37

Figure 14: Comparison of traftic rates at DIEC site.........................................38

Figure 15: Results from RSD simulatlons (LBL site)......................................39

Figure 16: Comparison of trafile rates at DLC and L.IIL sites.............................39

Figure 17: Intel IXP 1200 Areliliecture..........................................................4t

Figure 18: Results from Simulator............................................................46 


\section{CHAPTER 1: INTRODUCTION}

Denial-of-Service (DoS) attacks and Distributed Denial-of-Service (DDoS) are becoming increasingly prevalent in the Iniemet todny. A DoS attack is "characterized by an explicil attempt by altackers to prevent legitimate users of a service from using that service" [26]. In other words, a DoS altack aims to disrupt the nomal operation of an Internet service through malicious means, $A$ Distributed DoS attack is simply a DoS attack in which multiple distributed computers are used to perform the attack. The impact of these attacks can range from minor inconveniences, such as slow response from a website, to major financial consequences, such as the highly publicized DoS attacks in February of 2000. The latter atuacks targeted high-profile companies who rely on the intemet for their revenue, including Amazon.com. E`Trade, eBay, and Yahoo. Mlost recently. Microsoft. The SCO Group, and the RIAA lave been the target of DoS attacks. In the case of The SCO Group, the attneks even sueceeded in crippling thsir website.

Denial of Service attacks attempt to consume enough of a victim's limited resources until the victim ean no longer provide a certain level of service to legitimale users. These resources can be network bandwidth, computing cycles, or operating sysiem data strustures [2-1]. In order to consume these resources, allackers use two methods: software exploits and flooding attacks. Software exploits take advantage of bugs in the victim's operating system, and flooding attacks send a large amount of traffic to the victim. 
While most detected DoS atlacks have been shown to come from a single source [29], a growing trend is to utilize multiple sources to send attack traffic. This is called a Distributed DoS attack, and this thesis will concentrate solely on DDoS flooding-based attacks.

This thesis presents a new approach for the detection of DDoS SYN-flood attacks called Rotio-based SYN Flood Detection (RSD). RSD is a type of adaptive threshold algorithm and will dynamically adjust its beluvior to achieve optimal performance in the face of varying trafic conditions. A methodology is presented which optimizes RSD to make it more generally suited for widespread deployment, and its eflicacy is validated through trace-driven simulations. This thesis will also investigate the applicability of implementing RSD on a network processor and will show, through simulation, that a minimal amount of processing overhead is required to implement RSD.

This thesis is organized as follows: Chapter 2 provides background information on DDoS altacks and network processors as well as current research trends in both areas. Chapter 3 presents the RSD algorithm in detail, along with a methodology that can be used to optimize its performance. Chapter 4 analyzes the results obtained when an implementation of RSD is validated against a number of real-world traflic traces. Chapter 5 investigates the applicability of implementing RSD on a network processor. and Clapter 6 presents conclusions along with suggestions for future work. 


\section{CIAPTER 2: DACKGROUND AND RELATED WORKS}

This chapter will provide an understanding of different types of DDoS attacks and the mechanisms belind their efrectiveness. Existing solutions that attempt to prevent and detect DDoS attacks are presented, along with a background on network processors and current researcl in the area.

\section{I Types of DoS Attacls}

Denial of Service attacks attempt to consume enough of a victim's limited resoures until the vietim ean no longer provide a certain level of service to legitimate users. These resources can be network bandwidth, computing cycles, or operating system data structures [24]. In order to consume these resources, attackers use two methods: software exploits and flooding attacks.

Attacks that fall in the software exploit category typically take advantage of bugs in the victim's operating system. By taking advantage of these bugs, attackers can succeed in crashing or disabling the victim with a small number of packets. An example of this type of attack is the "ping-of-death" attack [28] which sends an extremely large ICMIP echo packet to the vietim and causes some operating systems to crash, reboot, or freeze. Another example is the "Land" attack [17] which sends a TCP SYN packet containing the vietim's IP as the source and destination address, thereby causing an infinite loop in the protocol stack [24]. These types of attacks can be mitigated through the use of patches and $O S$ updaies, and their prevalence has declined as users have become better about updating and protecting their systsms. 
A far more widespread and dangerous type of attack is a fooding-based attack [11]. Flooding allacks send a huge amount of traffic to the victim, resulting in bandwidth saturation of resource saluration as the victim attempts to deal with the traffic. The packets are typically generated to take advantage of the way a victim deals with certain prolocols, as is the case with SYN flooding [16] which exploits TCP's three-way handshake mechanism. Other examples include atlacks that use TCP packets with no flags set (TCP NUL.L attack), TCP packets with all flags set (Xmas altack), and packets with a non-exisien IP protocol number [24]. In all of these cases, the victim allocates resources to handle each packet, and the sheer number of packets causes the victim to crash.

\subsection{DDeS Flooding Attacks}

As mentioned before, a DDoS attack utilizes multiple hosts on the intemet to send a large volume of traffic to a victim, in the hopes of crippling it or making it unavailable. In general, there are two types of flooding attacks: direet attacks and reflector attacks [11].

\subsubsection{Direct Altacks}

A direet attack is when an altacker arranges to have a large number of packets sent directly to a victim. These packets can be generated solely by the attacker or. more commonly, through a DDoS attack network of compromised hosts. Such a network is composed of oae or more attacking hosis which control some number of masters, of controllers. These masters in tum control a large number of zombies, or daemons (figure 1) which are the entities that actually send the attack trafic to the victim. Attacking hosts originate the atteck sequence by notifying the masters who then instruct the zombies to 
begin sending altack packets. Attackers will typically compromise an attacking host first. which is then used to sean for other vulnerable hosts in order to install specific DDoS master and zombic programs.

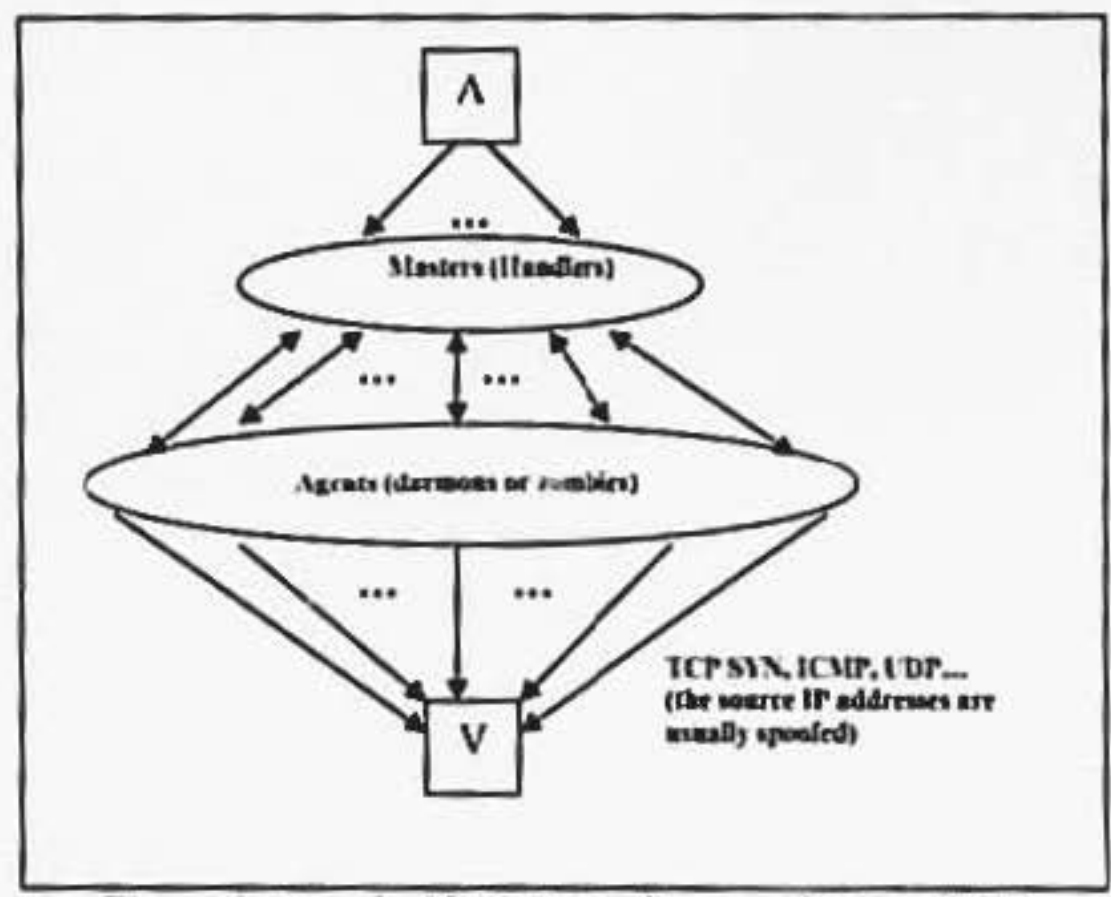

Figure 1: A typieal DDoS attack netwark (from |11)

Setting up a DDoS attack network gives the altacker the advantage of anonymity (since packets are being generated by other legitimale, albeit compromised, hosts) and inereased finepower due to the possibility of exponentinl growh using the master-zombie model. A number of tools are freely available on the Internet to aid in constructing an attack network and launching a DDoS attack, Including Trinoo, Tribe Flood Network 2000, and Stacheldraht [II]. For a detailed description of some of these tools and how to setup an altack network, see [29]. 
Most direet attacks use common Internet protocol packets, such as TCP, UDP, and ICMP. In the case of TCP, a popular attack is a SYN flooding attack [11][16] where a large number of TCP SYN packets are semt to a victim using a spoofed source IP address in the pocket. If the vietim is listening for connection requests, it will respond with a number of SYN-ACK packets and will then wait for a response. However, since the original TCP SYN packets have spoofed source IP addresses, these SYN-ACK packets will be sent elsewhere in the Internet and the victim will be lef waiting (figure 2n). A Lrge number of TCP SVN packets will create a large number of half-open connections on the victim, quickly consuming resources and preventing the victim from accepting legitimate connections or, in the worst case, crashing the victim. UDP and ICMP packets can also be used in a similar fashion to overwhelm the victim by causing the victim to send a large number of UDP or ICMP echo reply response packets to fake IP addresses.

\section{2,2 Rellector Attacks}

A reflector attack is one in which reflector nodes such as roulers or legitimate servers are used to lanch an attack. Reflector attacks are indinertly launched by an altacker and are good at hiding the identity of the antacker or increasing the distribution of an altack [30]. In a reflector attack, an attacker will send packets to the reflectors that require a response. such as TCP SYN packets (SYN-ACK nesponse) or ICMP echo request packets (ICMP echo reply response). However, these packets have the victim's IP address as the source IP address, causing the reflectors to send the response packets to the victim (figure 20). In this way. a large amount of traffic is unknowingly generaled by the reflectors and is targeted at the vietim, fooding the victim's link. 

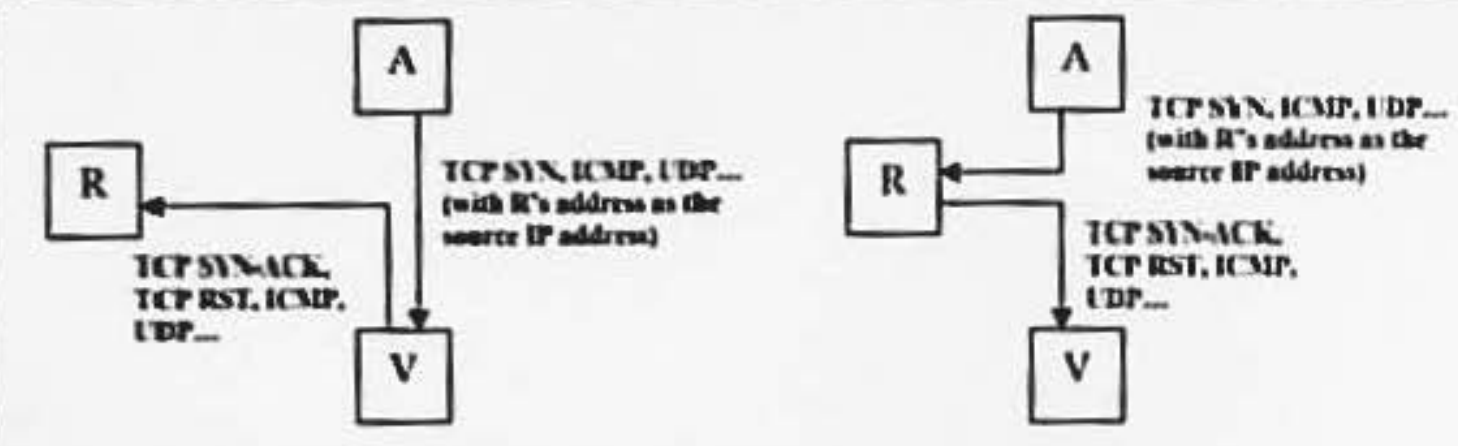

Figare 2: Flow of traflic in a direct atteck (a) and a reflector attack (b). Entity $A$ represents the attacker, $V$ is the vietim, $R$ is the reflector (from [11])

Reflector atuacks require the use of preselected reflectors and specific attack packets. Almost any protocol which employs an automatic response mechanism can be exploited in a reflector attack. In addition to TCP SYN packets and ICMP echo packets as mentioned above, ICMP error messages and DNS queries ean be used. For example. antack packets with invalid destination ports would cause a reflector to send ICMP port unreachable messages to a victim. Also, DNS can be exploited to take advantage of bandwidth amplifieation where a small number of attack packets (DNS recursive queries) can trigger langer packets in response (DNS replies) [31]. Table I summarizes some common reflestor antacks.

Because these reflected packets are generated by legitimate entities on the Internet. reflector attacks are more difficult to identify. Filiering based on spoofed IP addresses or route-based information becomes much less eflective. In addition, reflector attacks can be more distributed than direct atucks since attackers do not need to compromise hosts. Instead, they con utilize the many legitimate reflectors across the Internet. 


\begin{tabular}{|c|c|c|}
\hline Type of Attack & $\begin{array}{l}\text { Packets seat by an altacker to a reflector } \\
\text { (with a vietim's aduress as the souree } \\
\text { address) }\end{array}$ & $\begin{array}{l}\text { Pachets seal by the refiector to } \\
\text { tbe victim ta response }\end{array}$ \\
\hline Smart & $\begin{array}{l}\text { ICMIP echo queries to a suboet-directed } \\
\text { broodeasi addiens }\end{array}$ & ICMIP echo replies \\
\hline SYN Nooding & $\begin{array}{l}\text { TCP SVN packets to public TCP seners (c.g- } \\
\text { web scivers) }\end{array}$ & TCPSYN-ACK packets \\
\hline RST nooding & TCP packets to nonlistening TCP pons & TCP RST packets \\
\hline ICMP nooding & $\begin{array}{l}\text { - ICMP queries (usually echo queries) } \\
\text { - UDP pachers to nonlistening UDP } \\
\text { ports } \\
\text { - IP pachets with low TTL. values }\end{array}$ & $\begin{array}{l}\text { - ICMP replies (urually } \\
\text { echo replies) } \\
\text { - ICMP port unreacluable } \\
\text { measages } \\
\text { - ICAIP time excecded } \\
\text { mesiages }\end{array}$ \\
\hline DXS reply Rooding & DNS (recursive) queries to DNS senen & $\begin{array}{l}\text { DNS replies (usually much lasger } \\
\text { than DNS queries) }\end{array}$ \\
\hline
\end{tabular}

Table 1: Examples of common DDoS reflector attacks ([rom [II])

\subsection{Solutions to the DDoS Problem}

As DDoS atracks become more prevalent and more cosily, the need for solutions becomes vital. Generally speaking, there are three approaches towards the mitipation of DDoS attacks: attack prevention and preemption (prior to the attack), attack detection and filtering (during the altack), and attack souree traceback and identification (after the attack) [II].

\subsubsection{Attack Prevention and Preemption}

Attack prevention and preemption is probably the most diflicult of the three mitigation teclaniques. While this represents the first line of defense against DDoS attacks, the costbenefit ratio is extremely high for users who implement these techniques. Attack prevention requines the cooperation of individual hosis to protect their systems from contributing to a DDoS attack. These individual hosts can use monitoring tools and attack signatures to sean for attack traffic or known attack messages sent between masters and zombies. They can also take the necessary steps to protect their systems from 
becoming compromised and infected with a DDoS zombic process. However, most large ISPs and enterprise networks simply do not have the incentives to employ these difficult countermeasures [II]. Additionally, sinee the attack traflic is highly distributed amongst the sourees, the amount of atlack troficte is extremely low in relation to legitimate traffic. All of this combines to make altack prevention and preemption at the sources extremely tricky.

\subsubsection{Attack Deteetion and Filtering}

A second approach towards DDoS attack mitigation is attack detection and filtering. This approach attempts to identify an ongoing DDoS attack and then employs filters to block the attack trafic before it can reach the outside network.

A key consideration when dealing with attack detection is the placement of the detection and filtering mechanism in the network. Since DDoS attacks are highly distributed, the amount of attack traffic starts out relatively low at the source networks and becomes increasingly aggregated as it gets closer to the victim's network. Therefore, the eloser the detection mechanism is to the vietim"s network, the "easier" it is to successfully detect an altack. In contrast, the closer the detection meclanism is to the source networks, the harder it is to detect an attack. In the latter case, the ratio of attack traftic to legitimate traffic is very low, making detection diflicult companed to detection at the victim's network where the ratio is very high.

Unfortunately, the effectiveness of detection and filtering is also dependent on the loeation of the mechanism and is directly related to the difficulty of detection. By the 
time an altack has been detected at the victim's network, the incoming bandwidth has already been clogged by the attack packets and the altackers have succeeded in preventing legitimale users from aceessing the victim. The only real benefit to detection at the victim network is the prevention of a vietim crash due to resource starvation (since attack packets are dropped before reaching the vietim). Therefore, it would be much more effeetive to deteet an attack near the source network and drop the athack packets before they enter the Intemet, thereby saving Internet resources and preventing bandwidth starvation at the victim's network. However, as stated above, detection is more difficult as we move closer to the source networks. Therein lies the problem-as we move away from the victim's network, the effectiveness of detection and filtering increases, as does the difficulty. Figure 3 summarizes this relationship between detection effectiveness and difficulty.

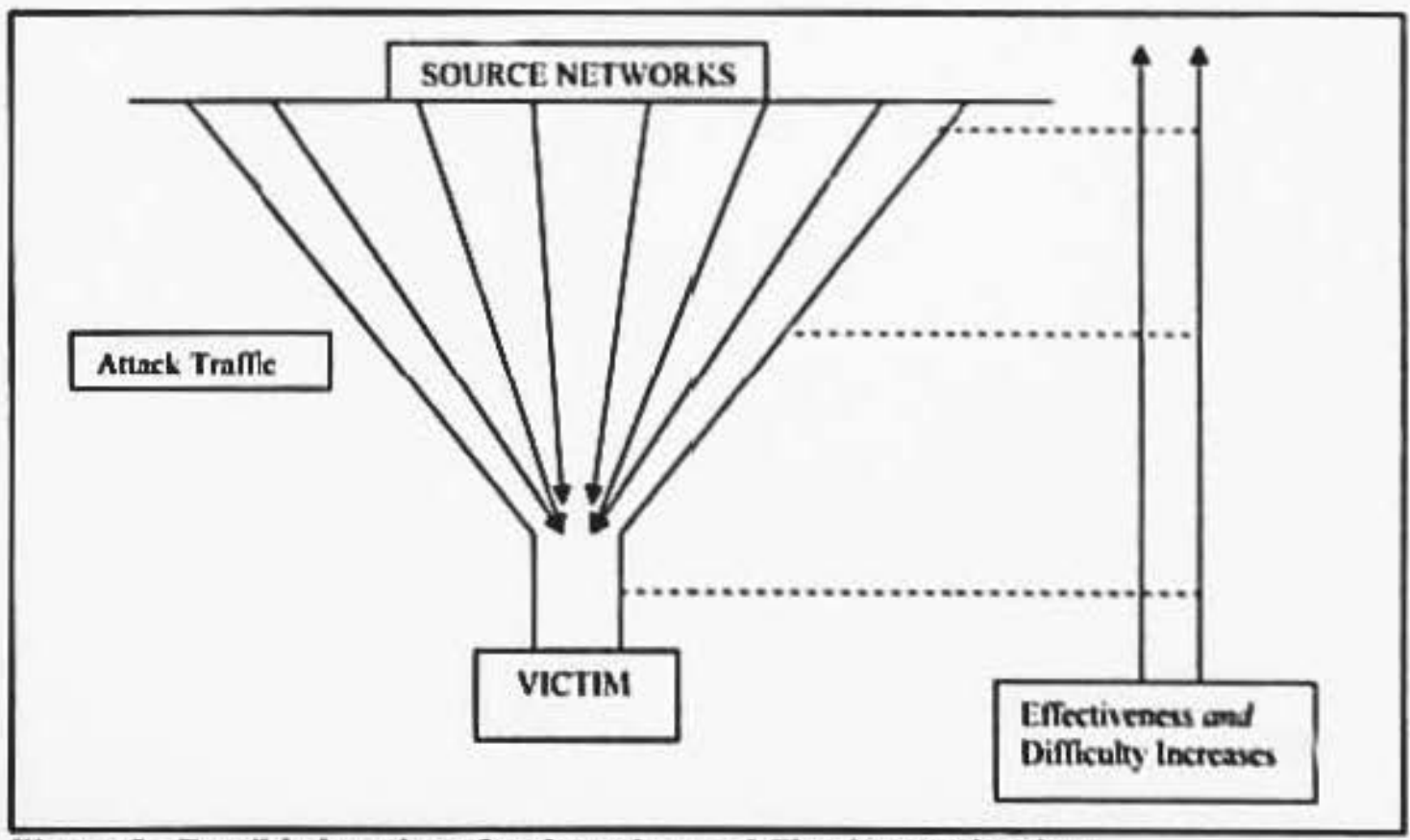

Figure 3. Posslble locations for delectlon and filtering meehantsm 
As pointed out above, is becomes casicr to deloc DDoS anteds whon the derection and filtering mechanism is located closer to the sictim's network. As a mesult, a number of commercial products have beea released which act as ivtersion detectirn drsibes and arre located at the firewall of the victim's sener [33]|341351]36]. However, the methods which these devices employ are proprictary, and litule infoemution is pithlidy anzilable. Also, they still do not address the problem of tinl handwidth sinuration. Therefore, mast of the current research is focused on the difficull problem of detection and filuering doact to the source netuorks.

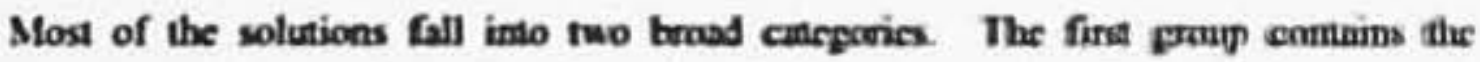

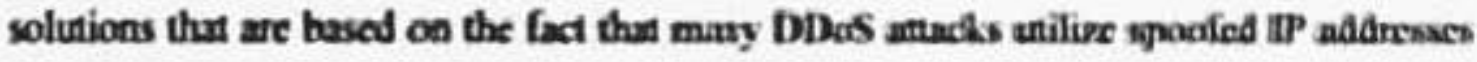
in the attack packets, and a varicty of methats are proposad to idemify thear mulicinus

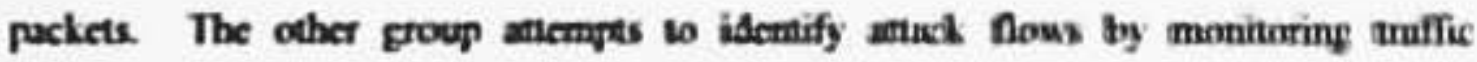
characteristics at various points in the notwoxk.

\subsubsection{Spoofed if Address Deteritem}

One of the simplest methods to thy and climinus spovied truffic is as perform neturit. ingress filtering $[17]$ at the source netwotk's ingress rounct of an the ISP. Huis simpily looks at the source IP aldress of all paclets emering the Imemet and onily allous packets with source IP addresses ther fall within the range of the sourse network as pass. This would effectively block most packets with emandomly spovfod If addrencex. Of courac. an attacker could always spoof IP adtresses thum fall within the runge of the snumer netwnih.

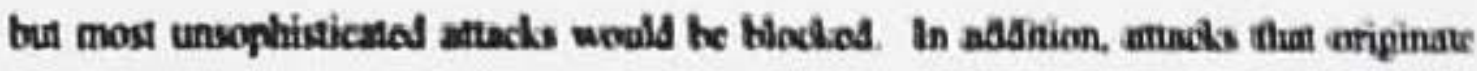
from compromised machines with valid If adtreses would puss unnrticed. While this 
seems like a relatively simple solution to the DDoS problem, actual implementation is difficult. In many large-seale networks, the overhead of inspecting every outgoing packet for source address validity is infeasible. Widespread deployment would also require the cooperation from all ISPs and network administrators, who see little benefit in implementing such an expensive feature in their edge routers.

Other solutions that are attempt to identify spoofed traffic include iSAVE [22][19] and Souree IP Address Monitoring (SIM) [12]|13]. iSAVE attempts to detect spoofed IP addresses on routers by building tables of incoming inierfaces and valid source IP addresses. The information contained in these tables are then shared between routers using iSAVE protocol messages and are used to filter out spoofed traflic. An implementation of the iSAVE protocol lus been demonstrated on the Intel IXP and has been shown to minimally impact routing performance. SIM. on the other hand, uses offline training to build IP Address Databases (IADs) which contain valid IP addresses seen at a network device. In the detection and learning phase, the amount of new IP addresses obsened is modeled using a change detection algorithm called Cumulative Sum algorithm [16][37]. An altack is detected when the change-point variable crosses sone Uhreshold. SIM has been shown to effectively identify DDoS attacks at the souree network using this scheme [13].

Park et. al. [15] take a different approach and attempt to eliminate spoofed IP addresses through the use of route-based distributed packet filtering (DPF). DPF uses routing information at network devices to determine if a packet arriving at a router is valid with 
respect to its souree and destination IP address fields. Park claims that if only $18 \%$ of autonomous sysiems implement DPf' on their border routers, a "synergistic filiering effect is achieved whose collective filtering action proactively prevents spoofed IP flows from reaching other autonomous systems in the first place" [15].

Jin et. al. [23] also take a unique approach by filtering packsts bascol on the TTL. field (hop-couni) in IP packets. The algorithm maintains a hash table containing address prefixes mapped to hop-counts and compares incoming IP packets against this table. Hop Count Filiering's (HCF) effectiveness relies on the assumption that attackers frequently choose some arbitrary value to place in the TTL. field when generating artack packets, and these packets with "invaliv" TTL fields can be easily identified. The aunhors have implemenied the scheme in the Linux kemel and kave shown a $90 \%$ success rate uith "little collateral damage" [23].

\subsubsection{Atonitoring Trafic Claracteristics}

The second group of solutions uses trafic monitoring and traffic characteristios to identify DDoS attacks [38][39]. D-WARD [18]|21] is detection mechanism that is placed at the souree network and identifies attack flows by monitoring two-way traffic flows between the network and the nest of the Internet. It works by looking for signs of communication difficulties and by comparing obsenved traffic statisties with prodefined models of normal traffic. When a possible DDoS attack is identified. D-WARD responts by raie-limiting the flow which can then confirm or refute the existence of an attack. 
Gil et. al. [14] proposes a scheme called MULTOPS which wilizes a unique tree-like data structure to characterize traffic characteristics on a network device. It looks for inconsistencies between traffic going to and from specific IP addresses. Specifically, if a certnin packet rate traveling to a host is higher than the returning packet rate, it is assumed that the destination host may be under attack and the outgoing traffic is then mate-limited. However, many flows on the Inlernet are asymmetric, which would increase the false positive rate of this scheme [12].

Wang et. al. [42] have proposed a mechanism to detect SYN floods at the source netuork called SYN-dog. The SYN-dog meelanism is localed on a leaf router and monitors the difference between the number of SYN and SYN-ACK packets. Its effectiveness is based on the strong correlation between SYN and SYN-ACK packets in a normal TCP session. Under normal conditions, a SYN packet will be accompanied by a responding SYN-ACK during a TCP connection setup. However, during a SYN flooding attack. large numbers of SYN packets with spoofed IP addresses are sent to a victim. This prevents SYN-ACK packets from retuming to the source network, and a discrepancy between SYN and SYN-ACK packets can be observed. SIN-dog views this difference between SYN and SYN-ACK packets as a "stationary, ergodic random process" [42], and models it as a Sequential Clange Delection [46] problem. SYN-dog normalires the difference using an estimated number of SYN-ACKs and then uses the non-parametric Cumulative Sum (CUSUM) method [47] to indicate possible attack-like traffic. For a detalled description of the SYN-dog algorithm, see [42]. 
Detecting DDoS attacks is a difficult task, especially when the goal is to detect aftack traflic as close to the souree as possible. Solutions must be easy to implement so that ISPs and network administrators will embrace enforcement while maintaining effective detection and low false-positive rates [40]. Researeh into detection and filtering is still being done, and novel solutions are still being proposed.

\subsubsection{Attack Source Traceback and Identification}

Attack source traceback, or IP traceback, is an after-the-fact solution to the DDoS problem. It atiempts to identify, or trace, the origin of attack packets in order to take future action against the attacker (or compromised machine). Unfortunately, it is infeasible to use IP traceback to stop an ongoing DDoS attack [II]. IP traceback solutions cannot always trace packets beyond a firewall or a NAT proxy, and it is useless ogainst DDoS reflector attacks since the packets are coming from legitimate network devices on the Iniernet. Nevertheless, IP traceback could be useful in some cases to identify certain attackers, allowing law enforcement agencies to take action. For a detailed survey on the state of IP traceback, see [41].

\subsection{Network Processon}

In any network infrastructure, routers play a key role by providing line-speed packet processing while supporting certain features including IP fonvarding. quality of service (QoS), and virtual privale networks. Many of these features go beyond a simple routing table lookup and require deep, stateful packet processing. The current trend in nextgeneration networks is to migrate these features towards the edges of service provider networks and away from the core routers, allowing the core to emeiently handle the high- 
speed switehing of large tramie aggregates. As a result, there is a need for flexibility at the edge routers as well as high performance to laandle packets at line-speed. Network processors (NPs) lave been developed to address this issue.

Traditionally. hardware-based design has focused on the use of Application-Specific Integrated Cireuits (ASICs) to perform most of the processing load. However, the long hardware development cycle and the lack of reprogrammability of these ASICs soon became an issue, especially with the need for flexibility in the rapidly tvolving network equipment market [1]. OMoading the processing laad to a general-purpose processor (GPP) gave designers plenty of flexibility, but the performance of these GPP's was inadequate. Accordingly, network processors were developed to provide the fexibility of a programmable architecture with performance approaching that of a hardware-based solution.

Typically, network processors consist of a set of programmable processors that are optimized for packet processing. They usually have specific instruction sets which allow for efficient packet landling, along with specialized coprocessors to of load common processing tasks such as table lookups or checksum computations. Because network processors must operate under strict performanee requirements, the entire design of the network proeessor (execution environment, memory, hardware accelerators bus aschitecture, etc.) has been optimized for high-speed packet processing. 
Network processor architectures fall into one of two models: serial or parallel. In the serial model (figure 4a), each core executes a small segment of the data-path code on every packet before sending it along to the next core for further processing. In the parallel model (figure 4 b), a number of network processor cores are present within the NP, and each core executes the entire data-path code on a single packet. The Intel IXP architecture [49] is based on the serial (pipelined) architecture and the IBM PowerNP [6] is based on the parallel model.

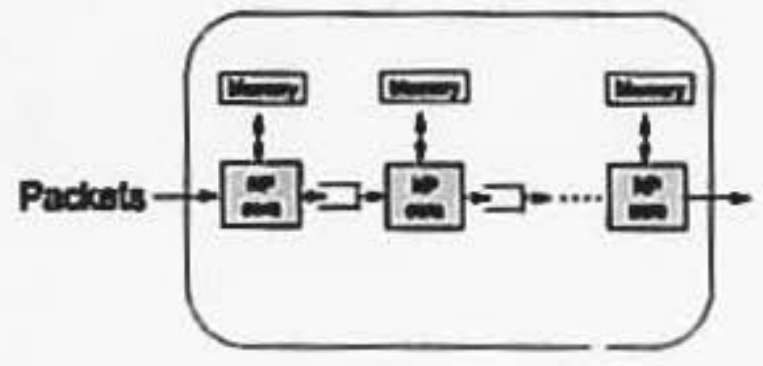

(a) Sortal modol

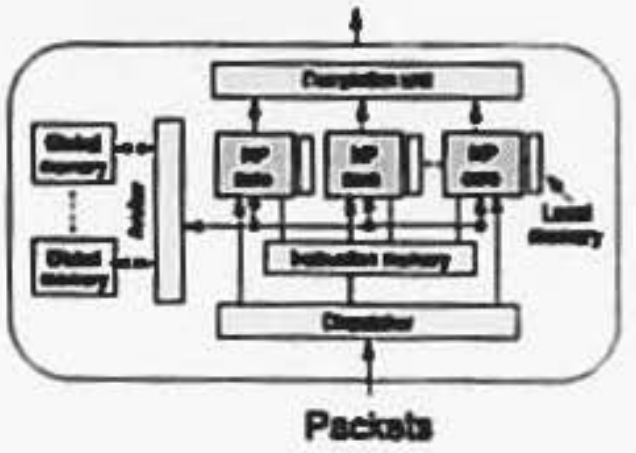

(b) Parallal model

Figure 4: Network processor architectures (from |II])

\subsection{Current Research on Network Processors}

Most of the network processor research today is being done using the Intel IXP arehitecture, specifieally the Iniel IXP 1200 [10]. Most of this research explores the performance and feasibility of porting existing sofluare-based rouler functionality (QDS. DimServ, eneryption/decryption, VPN, etc.) onto a network processor. For example, Lin et. al. [7] has implemented DinServ on the IXP 1200 with the aim of identifying bottlenecks within the IXP arehitecture and the DinServ algorithm. The authors found Uhat their Implementation could suppon Per-llop Fonvarding Beluavior (PHB) marking in 
Difiscrv at an aggregated throughput of $568 \mathrm{kbps}$, with the main bottleneck being the shared memory between the microengines (DRAM).

Burns ct. al. [8] implements audress assurance on the IXP I200 using the iSAVE protocol [22] and shows that the router suffers little to no performance degradation due to the added functionality. Spalink et. al. [3]|5] mrovides an in-depth analysis of basic IP forwarding functionality on the IXP 1200 and concludes that it can easily sustain line speeds for $8 \times 100$ Mbps Ethernet ports, leaving ample processing cyeles for additional packet processing. Similar to Lin et. al. [7]. the performance bottleneck was in the DRAM, and the authors estimate that a $26 \%$ improvement in line-speed processing is possible with faster memory.

Haas et. al. [I] discusses the suitability of network processors for "quality-of-senvice (active queue management and trafic engineering). header processing (GPRS turneling protocol), intelligent forwarding (load-balaneing without fow disruption), paylasd processing (active networks code interpretation and just-in-time compilation). and protosol stack termination (SCTP)" [1]. Still other areas of research include QaS [9]. active networks [10], packet classification [10], and various layer-4 applications such as web caching, load-balancing, and media streaming [10]. 


\section{CLIAPTER 3: RATIO-BASED SYN FLOOD DETECTION}

This section will provide an explanntion of SYN flooding, a common DDoS attack, and will also detail a proposed solution, dubbed malo-based SYN flood detection (RSD). It will also introduce a methodology for optimizing RSD based on traffic patterns of specific networks.

\subsection{SYN Flooding Altacks}

A SYN flooding aitack is so named due to its usage of TCP SYN packets as attack traffic. SYN flooding attacks fall into the flooding-based attack calegory and have become a popular weopon for DDoS attackers. In fact, it has been shown that more than $90 \%$ of DDoS attacks use TCP, and of these, SYN floods are the most prevalent igpe of attack [44].

SYN flooding attacks take advantage of TCP's three-way handshake mechanism in order to exhaust a vietim's resourees. In a normal TCP session, a server will listen for incorning SYN requesis on a well-known por. Once a connection request comes in from a client, the server will respond with a SYN-ACK packet and will establish a half-open connection. Once the server reeeives the ACK from the client, the three-way handshake is complete and the session can procecd (Figure 5). 


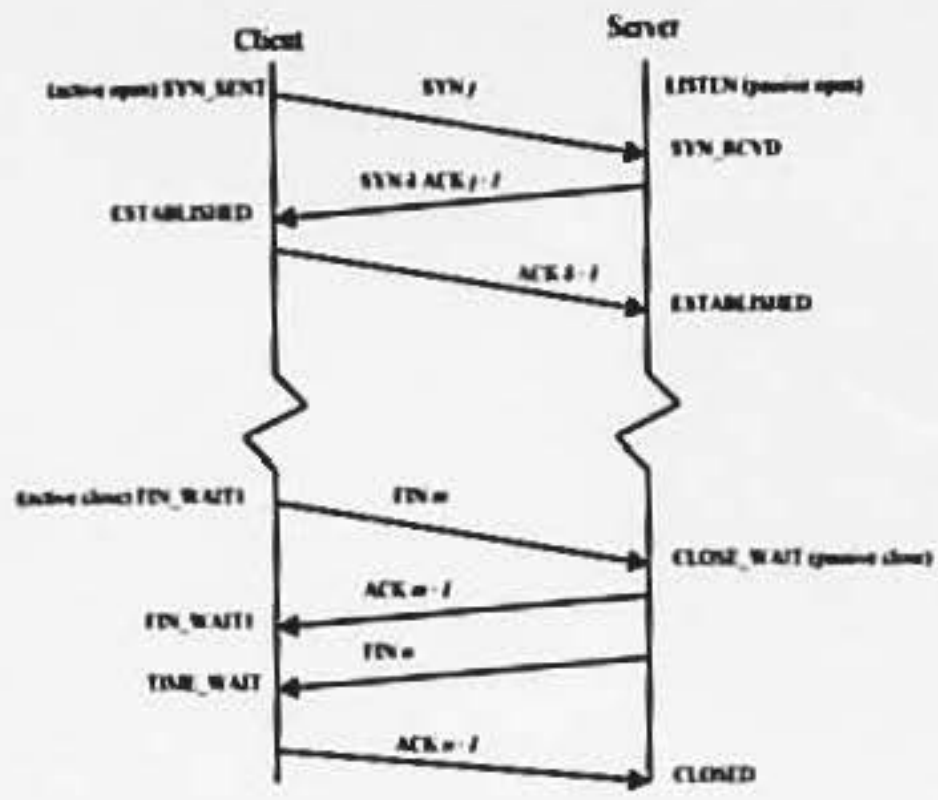

Figure 5: TCP states corresponding to normal conneetion establishment and teardown ([rom |45|)

In a SYN flooding altack, a large number of spoofed SYN packets with false source If addresses are sent to the vietim's TCP listening port. For each SYN packet, the victim will respond with SYN-ACK packet and will establish a half-open connection. However, since the source IP address was spoofed, these SYN-ACK packets will be sent to the spoofed IP address and will (presumably) be lost somewhere in the Internet. The victim. meanwhile, will continue to keep the lalf-open conneetion active for a period up to the TCP connection timeout and will not close the connection until two more SYN-ACK retransmissions have failed. This period of time typically lasts 75 seconds [42]. Since the victim only has a limited amount of memory to store these half-open connections, a large number of spoofel SYN packets will quickly fill up the queue and will prevent the vietim from servicing legitimate connection requests. 


\section{Ratio-hased SYN Flood Detection (RSD)}

Similar to the SYN-dog solution proposed by Wang et. al. [42], ratio-based SYN food detection (RSD) aims to detect SYN floods at the souree networks by monitoring the diserepancy between SYN and SYN-ACK packets at the edge router connecting a stub network to the Intemet. However, RSD uses an adaptive threshold based algorithm to detect an attack instead of a cumulative sum algorithm, as in [42]. The success of RSD is dependent on two key assumptions regarding a typical SYN flooding attack:

- The SYN packets have randomly spoofed source IP addresses.

- The randomly spoofed source IP addresses do not fall within the souree network subnet.

Under nomal traflic conditions, outgoing SYN packets will be accompanied by a responding SYN-ACK packet within one round-trip time (Figure 5). However, if the SYN packets lave spoofed source IP addresses, the victim server will respond to the spoofed address, and the RSD mechanism will never see these packets. Therefore, an incansistency between the number of SYN packets and the number of SYN-ACK packets would indicate a possible attack. Unfortunately, under nomal traffic conditions there is no perfect one-10-one correspondence between these packets. The most common reasons for this discrepancy are as follows [42].

- The TCP server receiving the SYN request is overloaded and is unable to reply with a SYN-ACK packet. 
- The link between the client and server is congested, causing the SYN request to be dropped. Since the TCP server never receives the SYN request, no SYN-ACK packet is generated.

As a result, RSD cannot detect a SYN flooding attack simply when a difference between the number of SYN and SYN- $A C K$ packets is observed. Instead, RSD monitors the ratio of SYN to SYN-ACK packets and sjgnals an altack when an abrupt change of this variable is observed, using an adaptive tureshold method based on previous ratio measurements. Similarly, SYN-dog [42] monitors the normalized difference between SYN and SYN-ACK packets and uses a non-parametric Cumulative Sum (CUSUM) method [47] to indicate an altack.

Under typical conditions, the number of SYN and SYN-ACK packets is strongly correlated, independent of sample time, site, and time-of-day. Traffic traces gathered at a stub network's connection to the Intemet were obtained and analyzed, with the results shown in figure 6. Figure Ga represents a one hour trace of all traffic between the Digital Equipment Corporation and the Internet on March 8, 1995. Figure 6b represents a one hour trace of all trafic between the Lawrence Berkeley Laboratory and the Imemet on January 21. 1994. Note that there is a strong correlation between SYN and SIN-ACK packe1s, but there is no one-10-one relationship. 


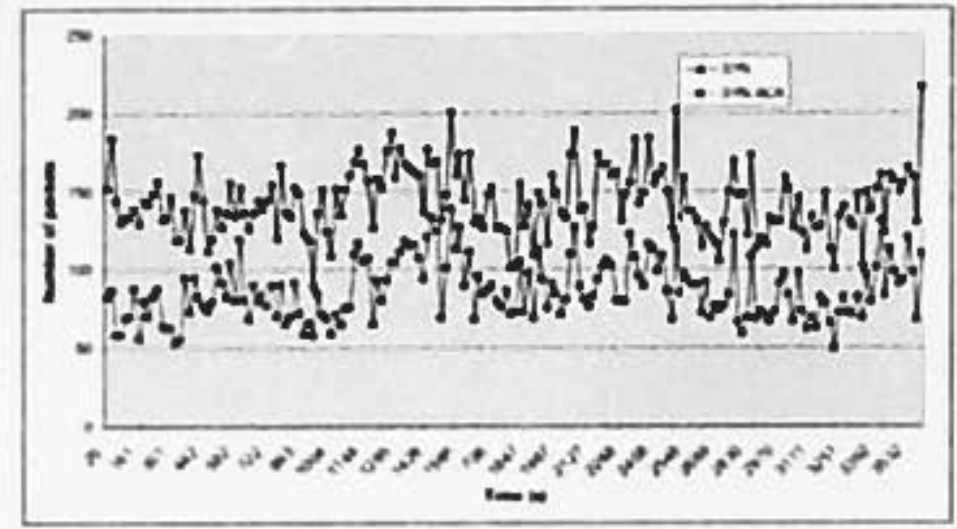

(a)

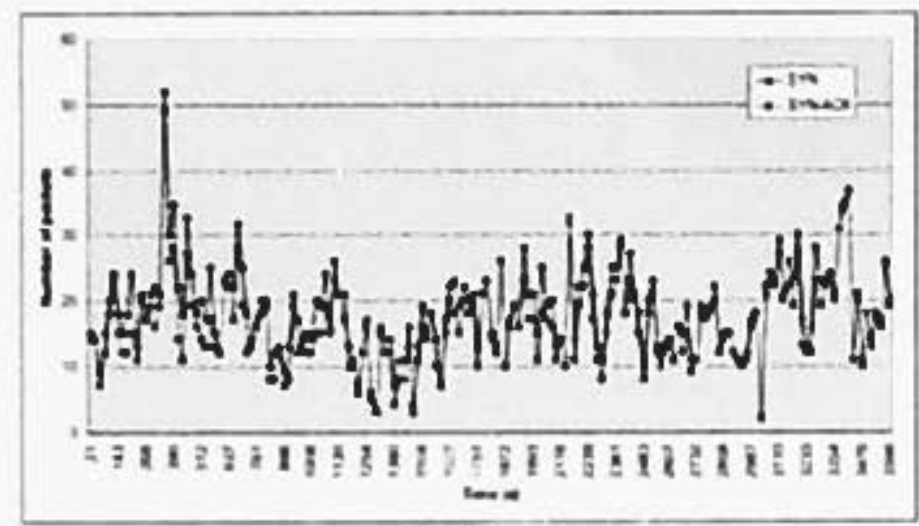

(b)

Figure 6: SYN and SYN-ACK packet counts at DEC(a) and L.BL(b)

During an attack. spoofed SYN packets will be observed at the leaf muter but the number of SYN-ACK packets will remain relatively unclanged. since the vicim will be sending the responding SYN-ACK packets to the spoofed IP address. As a result. the differente between SYN and SYN-ACK packets will increase, indicating a possible attack. However, we cannot infer an altack by simply using the difference as our measurement. Intemet traffic will sary depending on a number of factors, including time of day and type of users, and a normal increase in traflic load may also lead to an increase in the difference between SYN and SYN-ACK packets. For example, assume one out of every three TCP connections requires a retransmission of the beginning SYN packel due to one 
of the tho factors mentioned earlier. If an edge router sees $30 \mathrm{new}$ TCP connections every minute, then the router will see 10 more SYN packets than SYN-ACK packets every minute. However, if an edge router experiences a bursl of high traffic and there are 60 new TCP connections every minute, then the difference between SYN and SYN-ACK packets will be 20. Obviously, the increase in the difference does not indieate an atlack, but is a direct result of varying traffic patterns.

In order to make artack detection less sensitive to varying traffic patters, RSD monitors the ratio between SYN and SYN-ACK packets at the edge rouler. The algorithm for detecting an attack is relatively strightforward and is a type of adaptive threshold algorithm. It tests whether a given traffic measurement, the ratio of SYN to SYN-ACK packets in our case, exceeds a set threshold in a certain time interval. The threshold value is calculated using recent traflic measurements to make the algorithm applicable to differing traflic conditions.

\subsection{RSD Detnils}

Let

$n=$ some time interval $A t$

and let

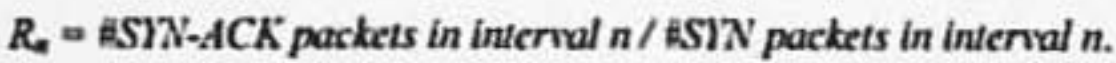

Then $R_{*}<1$ and should be close to $I$ due to the correlation of SYN and SYN-ACK packets. When an attack oceurs, the number of SYN packets will increase while the number of SYN-ACK packets will stay the same, resulting in a decrease of $R_{*}$. During 
the detection plase, RSD calculates $R_{a}$ for every time interval $\Delta t$ and signals an altack when

$R_{a} \leq\left(a \cdot D_{a-1}\right)$

Where $a$ is some value between 0 and $I$ and is our threshold variable and $O_{m-1}$ is an approximate mean value of the past $n-l$ measurements of $R$. $D_{n}$ is calculated using an exponentially weighted moving avenge function

$O_{a}=\beta\left(O_{m-1}\right)+(1-\beta) R_{m}$

where $0<\beta<1$ is the exponentially weighted moving average factor.

The tunable variables in this algotithm include $a, \beta$, and $\Delta t$. The following sections discuss the effects of altering these variables.

\subsubsection{Varying $a$}

a represents the threshold level for indicating a DDoS attack. By increasing $a$, RSD becomes more sensitive to small fluctuations in $R_{\infty}$ and results in a higher probability of attack detection along with a higher number of false positives. By decreasing $a$, RSD becomes more immune to false positives but only signals altacks if the SYN flood rate is very high, thereby decreasing its effectiveness. Through initial trial-und-error, $a$ is set to 0.7 in RSD in an attempt to balance a low false positive rate with a high degree of effectiveness. 


\subsection{Varging $\beta$}

$\beta$ represents the exponentially weighted moving average factor and determines how much weight is given to past measurements compared to the current measurement when calculating the moving average. A high value of $\rho$ smoothes out the moving average $O_{\text {a }}$ and makes it less susceptible to abnormal measurements of $R_{*}$. A low value of $\beta$ will put

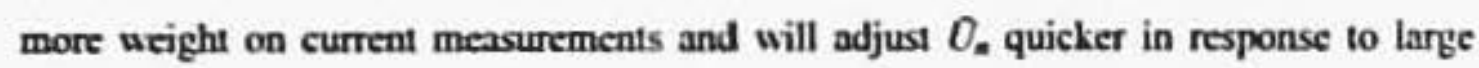
changes in $R_{\text {en }}$. Since intemet traffic is typically very bursty on a shon timeseale and slowly varying on a long timescale, $\beta$ is initially set to 0.9 in RSD.

\section{3 .3 Varying $\Delta t$}

At represents the time interval (in seconds) that RSD will use when detecting SYN flooding attacks. It also represents the minimal attack detection time, as RSD can only flag an artack after each time interval is complete. As such, determining an optimal value for $\Delta t$ is very important for good performance of RSD. If $\Delta t$ is too small, RSD will not see a significamt number of $\mathrm{SYN}$ and $\mathrm{SY}$. . CK packets during each interval, resulting in a high false positive ratio (FPR. or "Hfalse pasitives in $n$ intenvals $/ n$ ) and poor performance. As $A t$ increases, the FPR decreases at the expense of attack detection time. Ideally. we would like for RSD to be able to detect SYN flooding attacks in the shortest tiro possible with a FPR of 0 . but clearly a tradeoff exists between these two goals. The next section will explore this tradeoff in greater detail.

\subsubsection{RSD Training Mlodule}

An optimal value of $\mathrm{dt}$ will result in quick attack detection time with the lowest false positive rate (FPR) possible. Determining a good value for $\Delta t$ is also very dependent on 
the traflic patterns, even more so than $a$ and $\beta$. For instance, a good $A t$ for a large stub network may result in extremely poor performance in a smaller stub network, as $R_{n}$ can vary wildly when $\Delta t$ is too small to accumulate an adequate number of SYN and SYN. ACK packets in the time interval. This same problem can occur on the same stub network when traflic patterns sary due to time of day fluctuations. As a result, a training module is needed to customize $A t$ for successful application in a specific network environment. This training phase is done ofmine and will result in an optimal $\mathbf{d t}$.

Omine training is done with a typical traffic trace obtained at the edge router where RSD will be deployed. It is assumed that this trace does not contain any SYN flood attacks. This is important, as it implies that any atlacks cauglat by RSD can be considered false positives. Through simulation, different values of $d t$ can be set in RSD and the algoritlm can be applied multiple times to the trafic trace, each time calculating the false positive matio (FPR). Based on these simulations, an optimal value of $d t$ is then chosen. The results of applying RSD on the DEC and LBL traces with $A t$ varying between 1 and 30 seconds is shown in figure 7 below. 


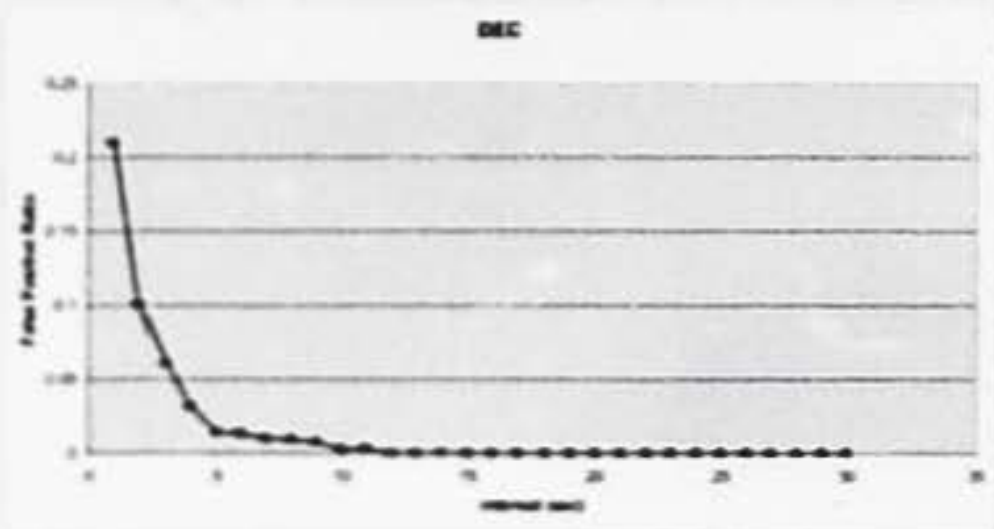

(a)

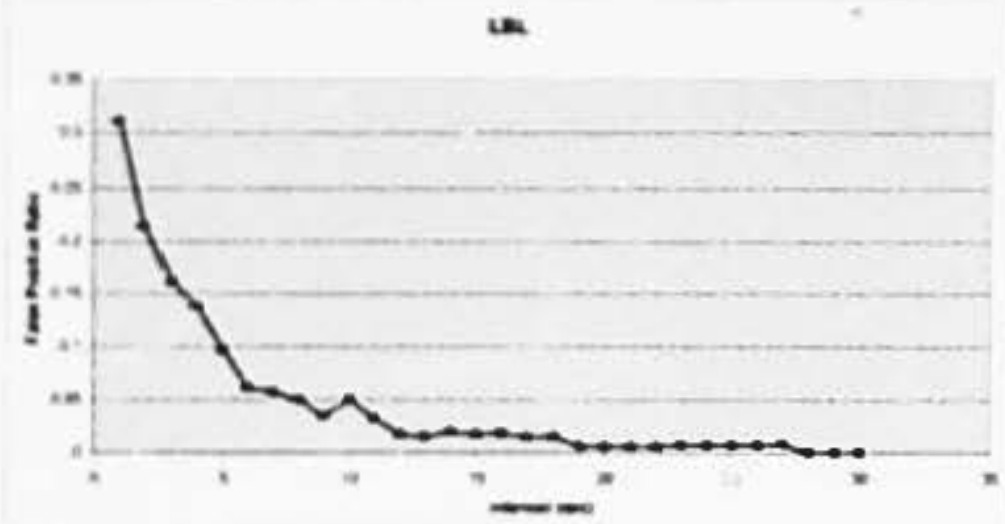

(b)

Figure 7: Ellects of varying $\Delta /$ at DEC(a) and LBL(b)

Clearly. there is am exponential relationship between the interval $(\Delta)$ and the FPR. Since we are interested in a low FPR with minimal detection time, we call see that a good $\Delta$ value is 10 seconds for the DEC netuork and 20 for the LBL network. The fiscrepancy between these tuo values can be confirmed by observing figure 6 and noting the difference in SYN and SYN-ACK traffic. The DEC trace has much more SYN and STN-ACK traffic, and will therefore show less variation of $R_{\infty}$ as smaller intervals are used. resulting in a loser FPR for the same value of $\Delta /$ as in the LBL trace. 


\subsubsection{Dyaamically Adjusting $\Delta$}

A key feature of RSD is the ability to atapt to changing traflic conditions. This is achieved by varying $\Delta$ dynamically, in order to achicve our goal of fast attack detection (low $\Delta$ ) with a low FPR. As mentioned earlier, trafic conditions may fluctuate due to factors such as time of day or type of user, and as a result $\Delta \mathrm{s}$ may become less than optimal. To achieve adaptability, RSD first calculales a new value, \#SA, for every differen $\Delta$ used in the training plase. $\forall S A$ is the exponentially weighted moving average of the number of SYN-ACK packets in each $\Delta$. Intuitively, it represents the typical amount of SYN-ACK troflic in each $\Delta$. Figure 8 shows $\forall S A$ salues when $\Delta$ is saried from I to 30 seconds on the DEC trace. As expected, when we increase $\Delta$, more SYN-ACK packets are observed per time intenal.

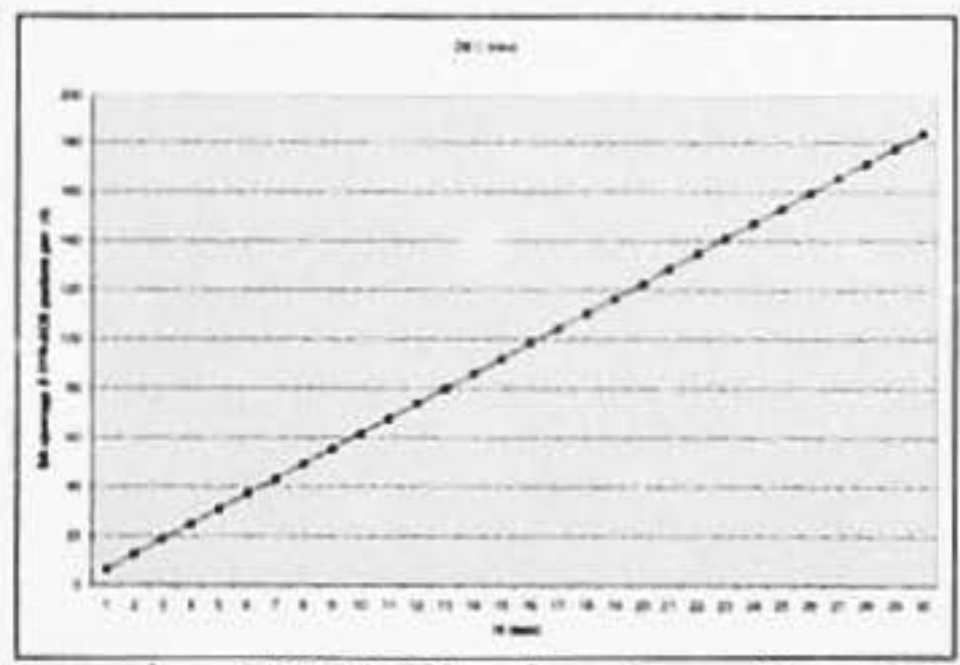

Figure 8: Average number of SVN-ACK packets oiseried in each $\Delta t$ for DEC trace

After an optimal $\Delta$ is chosen (10 seconds in the case of the DEC trace). RSD saves the

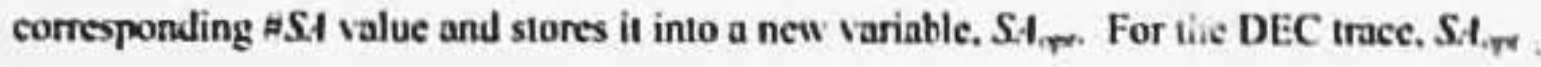
$=61 \mathrm{SYN}-\mathrm{ACK}$ packets. It is important to remember that $S \mathrm{I}_{\text {ap }}$ is set afler the training plase is completed and is not clanged during normal operation of RSD. 
During the detection phase, RSD compares $S \mathrm{I}_{\text {ap }}$ to the observed number of SYN-ACK packets $(\#, i d)$ in each $\Delta t$. The following pseudo code illustrates the belavior of RSD during each time interval:

If $a S i<S I_{\text {cre }}$ then

Increase $d i$ by $2 x$ seconds

Else if $i S: 1>S \cdot f_{\text {op }}$ then

Decrease di by $x$ seconds

Where $x$ can be varied depending on how quickly we would like RSD to adapt to changing traflic conditions. $x$ is set to I second in this implementation in order to keep At stuble over the long run.

Using this simple test at each time interval allows RSD to adjust Af dynamically in order to keep it close to optimal. For example, if a reduction in traflic is obsenved by RSD (indicated by a reduction in the number of SYN-ACK packets), then RSD will increase At in order to increase the number of SYN-ACK packets measured per time intenal. This will keep the FPR from becoming too large. Concurently, if an increase in traflic is observed then RSD will decrease At in order to keep our detection time as small as possible. 
To show the effect of varying trafic conditions on the FPR if we were to use a static value of $\mathbf{M}$, another trace collected at the DEC site was run through the training phase. This trace, tabeled DEC-2, was gathered four hours prior to the original DEC trace (labeled DEC-1). Figure 9 shows the effects of varying $\Delta /$ on both the DEC-1 and DEC-2 traces.

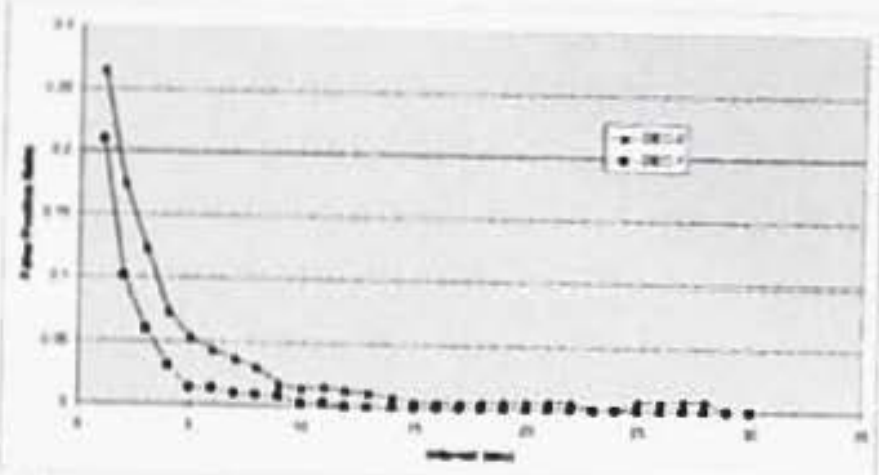

Figure 9: Efreets of varying $A /$ on DEC-I and DEC-2

From figure 9, we can see that the optimal $\Delta /$ for DEC-2 will be longer than the optimal $\triangle 4$ for DEC-1. This is due to the lower amount of SYN-ACK traflic in the DEC-2 trace. as shown in figure 10.

Figure 10: Average number of SYN-ACK packets observed in each $A$ for DEC-1

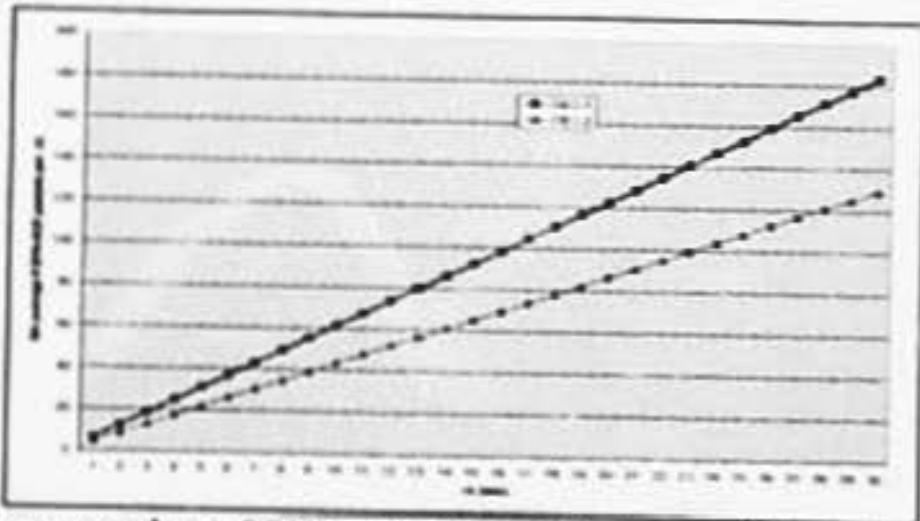
and DEC-2 
value of $\Delta t$, another trace collected at the DEC site was run through the training phase.

This trace, labeled DEC-2, was gathered four hours prior to the original DEC trace (labeled DEC-1). Figure 9 shows the effects of varying $\Delta t$ on both the DEC-I and DEC-2 traces.

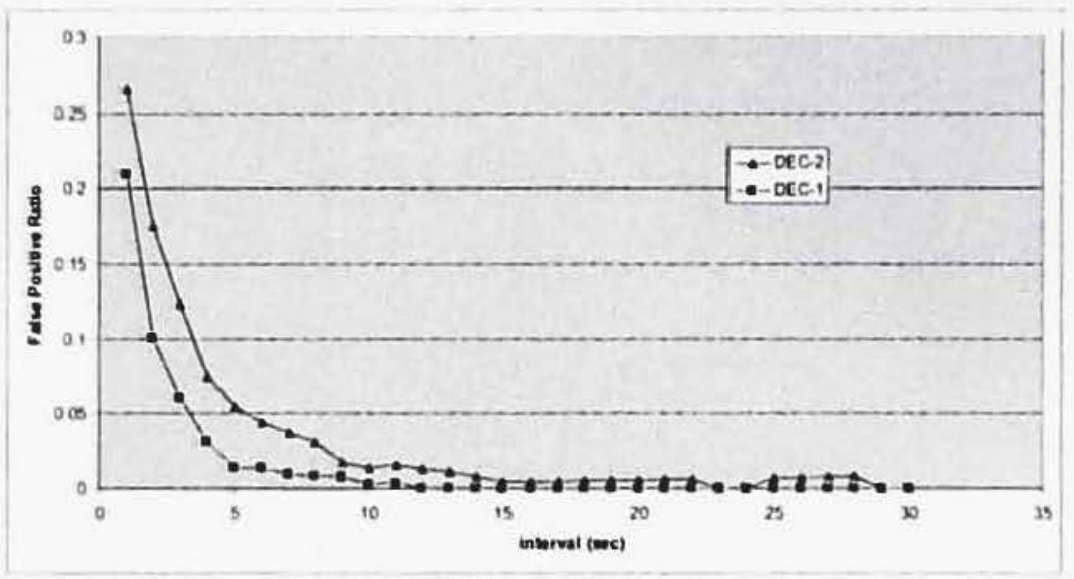

Figure 9: Effects of varying $\Delta t$ on DEC-1 and DEC-2

From figure 9, we can see that the optimal $\Delta t$ for DEC-2 will be longer than the optimal $\Delta t$ for DEC-1. This is due to the lower amount of SYN-ACK traffic in the DEC-2 trace, as shown in figure 10.

$$
{ }^{\infty} 1
$$


From figure 9, we can see that the optimal $\Delta t$ for DEC-2 will be longer than the optimal $\Delta t$ for DEC-1. This is due to the lower amount of SYN-ACK traffic in the DEC-2 trace, as shown in figure 10.

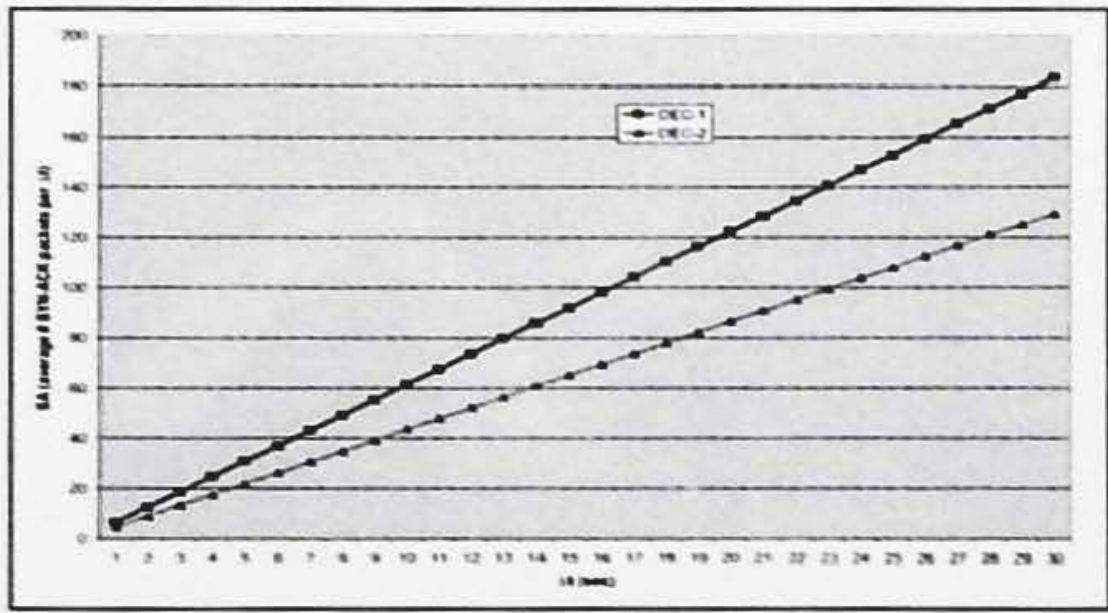

Figure 10: Average number of SYN-ACK packets observed in each $\Delta t$ for DEC-1 and DEC-2 
Clearly, if we lave trained RSD on the DEE-1 trace, then a static value of At (10 seconds) will result in a higher FPR when traffic conditions such as in DEC-2 are present. Therefore, in the ease of decreasing traflic load (i.e. DEC-1 to DEC-2), RSD will increase the time interval in order to ensure a low FPR. In contrast, when the traffic load is increasing (i.c. DEC-2 to DEC-1), RSD will decrease the time interval, which will result in a lower attack detection time. Since maintaining a low IPRR is more important than ensuring the lowest possible detection time, RSD will increase At by $2 x$ instead of $x$. This will keep At slightly larger than necessary in the long run. 


\section{CHAPTER \&: RSD IMPLEMENTATION AND RESULTS}

In order to investigate the efficacy of RSD, a number of trace-driven simulations were performed. Traffic traces were obtained from the link connecting two siles to the Imernet: the Digital Equipment Corporation and the Lowrence Berkeley Loboratory [48].

Table 2 summarizes the pertinent information regarding the collected traces.

\begin{tabular}{|c|c|c|c|c|}
\hline TRACE NAMIE & LOCATION & DURATION (min) & DATE COLECTED & START TIMIE \\
\hline DEC-PKT-1 & $\overline{D E C}$ & 60 & Mlarch E. 1993 & 2200 \\
\hline DEC-PKT-2 & DEC & 60 & Alach 9, 1995 & $02: 00$ \\
\hline DEC-PKT-3 & DEC & $\infty$ & Atarch 9.1993 & 10.00 \\
\hline LEL-PKTA & Lil. & 60 & Ionary 21. 1994 & 14.00 \\
\hline LEL-PKT-5 & LBL & 60 & Lanum 28,199 & 14.00 \\
\hline
\end{tabular}

Table 2: Summary of trafic traces used in simulations (from [48])

Following the methodology presented in the previous section, the RSD training phase was performed for each of the individual traces to determine optimal $d t$ and $S t_{\text {qF }}$ values. Figure 11 shous the results of this training phase, and table 3 shows the resulting optimal values chosen for each respective trace. 


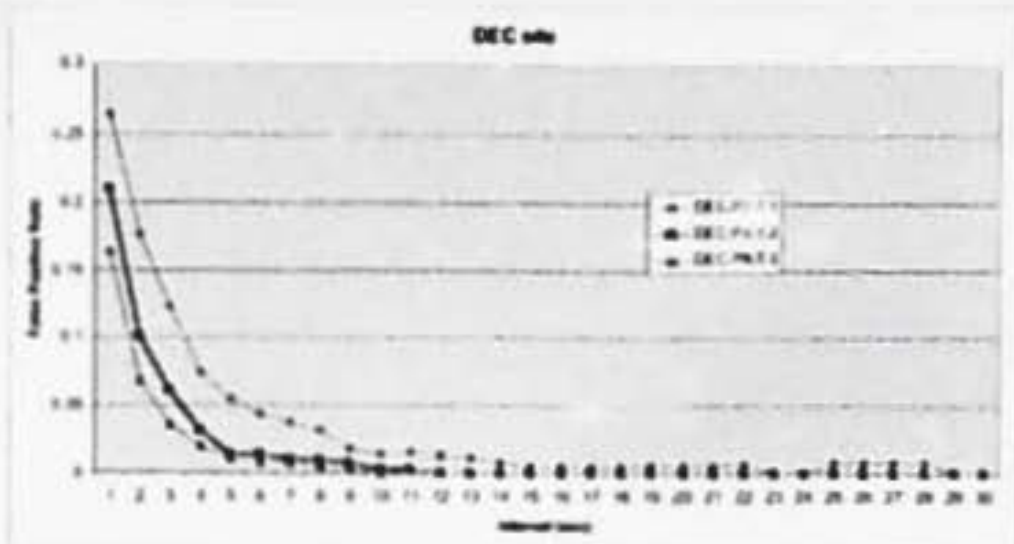

(a)

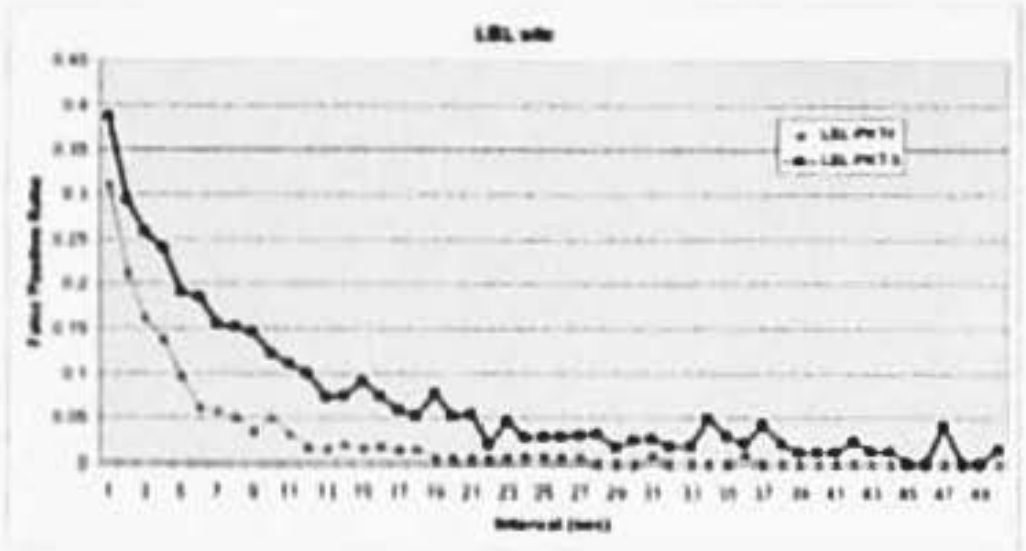

(b)

Figure 11: Results from RSD tralning phase an DEC (a) and L.BL (b) traces

\begin{tabular}{|c|c|c|}
\hline TRICENAIIE & OrTISAL $\mathbf{v}$ (nx) & S.t. ipackete per $\mathbf{S}^{\prime}$ \\
\hline DEC.PKT-1 & 23 & क) \\
\hline DEC-PKT:2 & 15 & 91 \\
\hline DEC-PKT-3 & 10 & 102 \\
\hline UIL-PKT-4 & 30 & 25 \\
\hline UIL-FKT-5 & 45 & $2 x$ \\
\hline
\end{tabular}

Tabte 3: Optimal values for RSD for diflerent iraces

Table 3 illustrates the importance of the training plase for optimal performance of RSD. Initial values for $\boldsymbol{V}$ ean range from very short intervals, as in the DEC.PKT-3 trace (10 sec). to long intervals, as in the LBL-PKT-5 (45 sec). Clearly, a static value for $\mathrm{A}$ will not provide optimal performance for varying trafle conditions and may result in a high FPR or reduced artack detection time. depending on conditions. 
I is also importan to note the correlation between optimal values of $\mathbf{S i}$ epe within each site. Recall tha RSD will dynamically vary $\Delta t$ based on the number of SYN-ACK poekets observed per time interval (ESA). In effect, RSD is adjusting At in order to keep

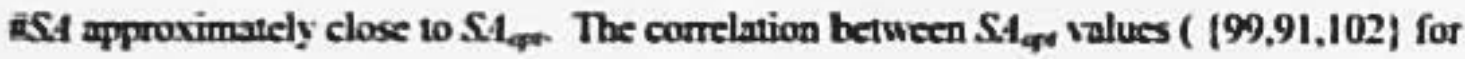
the DEC site, (2528) for the LBL site) shous that RSD will provide optimal detection even in the face of vaying trafic conditions. For example, assume that RSD is tmined on DEC-PKT-1 and begins the detection phase with $\Delta t=23$ and $S A_{\text {qut }}=99$. If traffic conditions change to those similar in DEC-PKT-2 suddenly At becomes less than optimal. In this case, RSD will see that eSA $>S A_{\text {qn }}$ and will decrease $A t$ until $i S A$ approaches $S A_{\text {or }}$ resulting in an optimal $A t$ (uhich is 15 in this case).

To verify this observation, the RSD training phase was run on DEC-PKT-1 and the variables were used uhen running simulations on DEC-PKT-2 $\left(4 t=23\right.$ and $\left.S A_{\text {qq }}=99\right)$. As expected. RSD dynamically adjusted $\Delta t$ by reducing it since a $\Delta t$ value of 23 resulted in E⿺辶t being greater than $S t_{\text {qpe }}$ At was observed to approach its optimal value of 15 seconds in approximstely 10 intervals. The correlation between $S A_{\text {re }}$ values within the DEC sile cnsured that the dynamic behasior of RSD kept At optimal.

Orace the training phase was complete, simulated artacks were inserted into the traces and the RSD algorithm's attack detection capability was explored. All attacks began 10 mimutes into the trace and lasted for 10 minutes. According to [44], this is the typical 
antack duration abserved in the Internet. The SYN flooding rale was saried from 1 to 20 SYN arrack packets per second and these traces were run through the RSD algorithm.

\subsection{DEC vite results}

Figure 12 below shous the results of varying the SYN flooding rate in the DEC traces and the ability of the RSD algorithm to correctly identify an antack condition. The x-axis represents differiag SYN attack flooding rales (1-20 SYN packets per second). The yaxis represents the ratio of detected antack intervals over the total number of aftack interials. Recall that all simulated anacks last for 10 minutes, thus in the case of DEC. PKT-1 where $\Delta$ is initially set to 23 secondx, there will be approximately 26 attack interials. It is clear that an increase in the SYN flooding rate results in RSD correctly identifying a higher percentage of the intervals which contain an attack. For example, in the ease of DEC-PKT-1. RSD was able to detect $100^{\circ}$. of the attack interials 126 out of 26 imervals) when the SYN flood rate reached 6 SYN packets per second. In contrast. RSD was unable to detect an atack (0 out of 60 intervals) when a rate of less than 10 SYN packets per second was inserted into DEC-PKT-3.

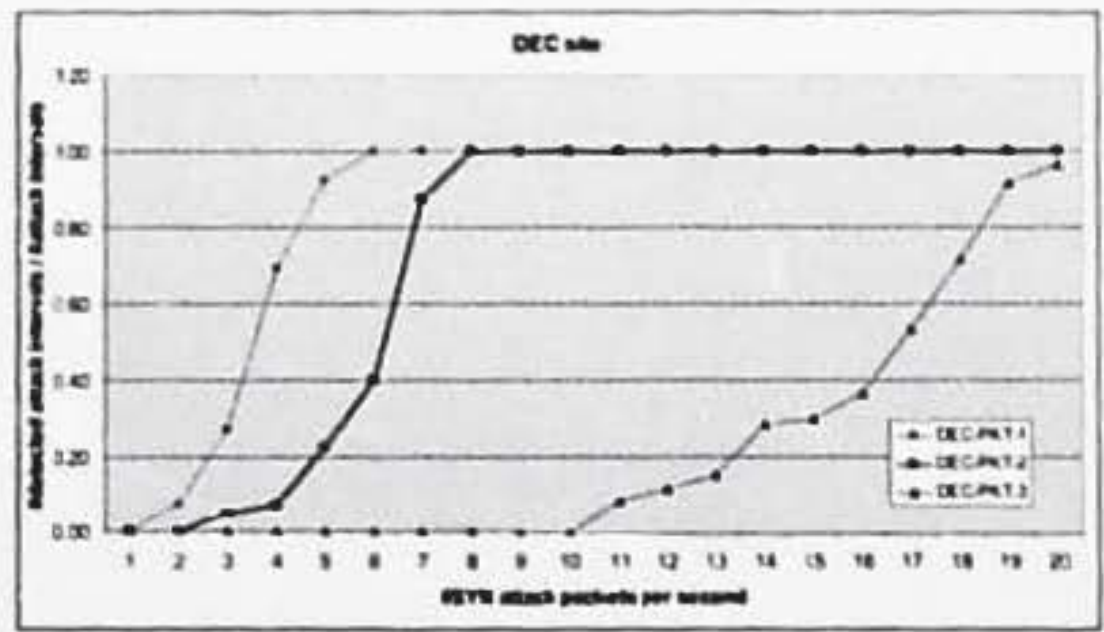

Figure 12: Results from RSD simulations (DEC site) 
the case of Uex -17N1-1, nue ............

26 intervals) when the SYN flood rate reached $6 \mathrm{SYN}$ packets per second. In contrast,

RSD was unable to detect an attack ( 0 out of 60 intervals) when a rate of less than 10

SYN packets per second was inserted into DEC-PKT-3.

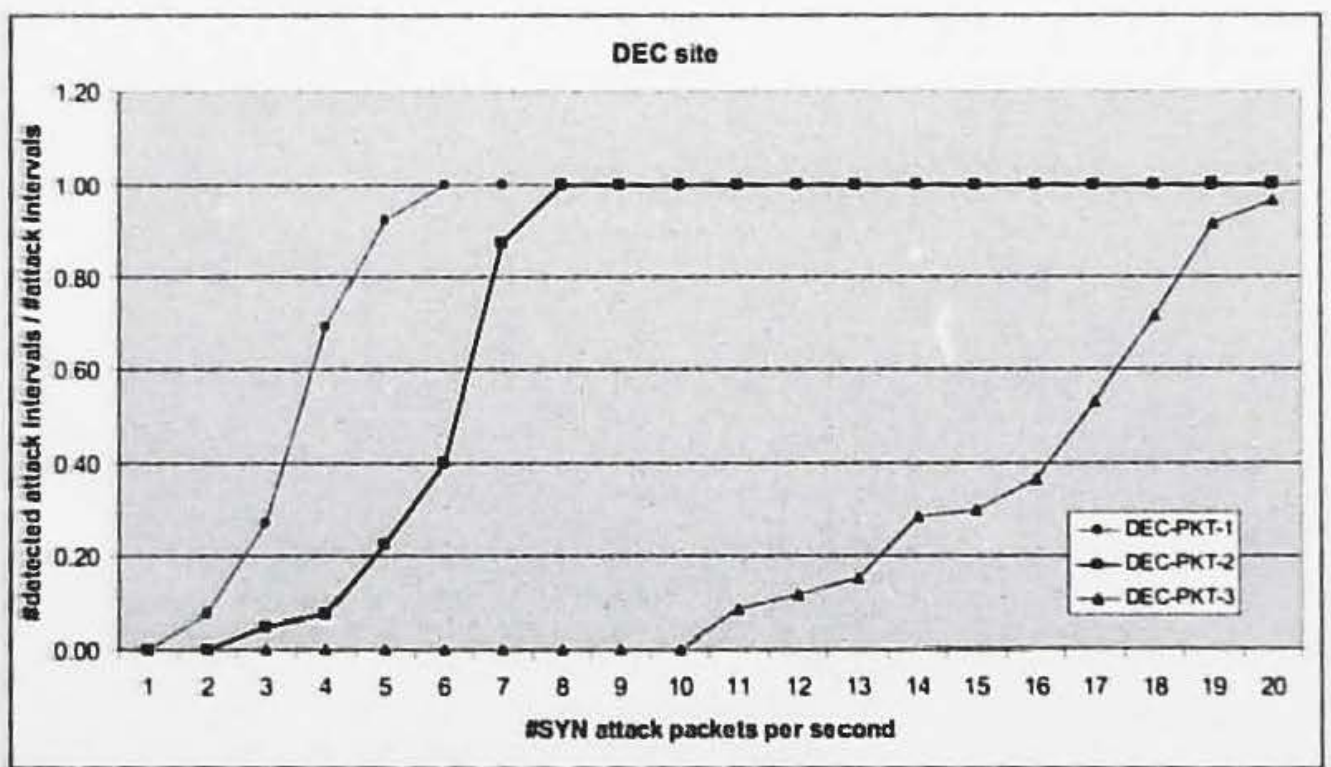

Figure 12: Results from RSD simulations (DEC site) 


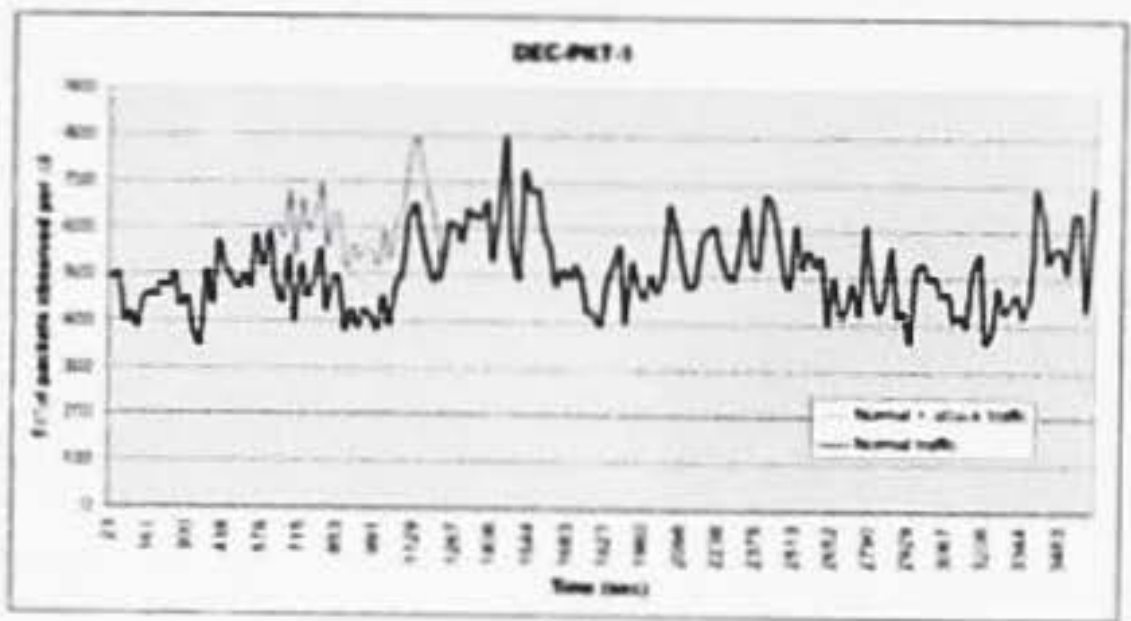

(a)

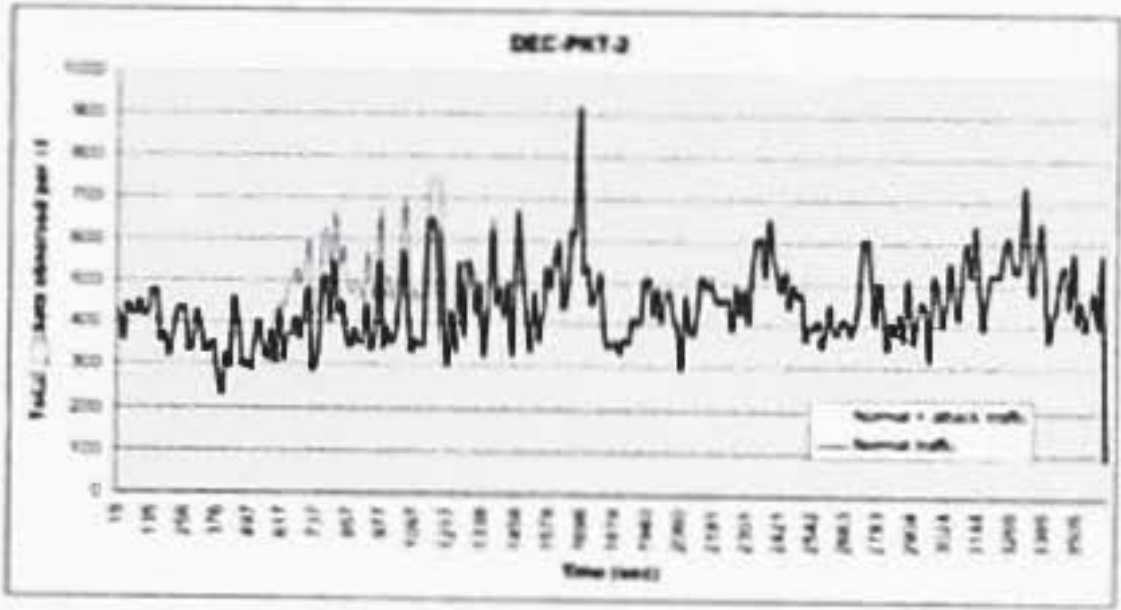

(b)

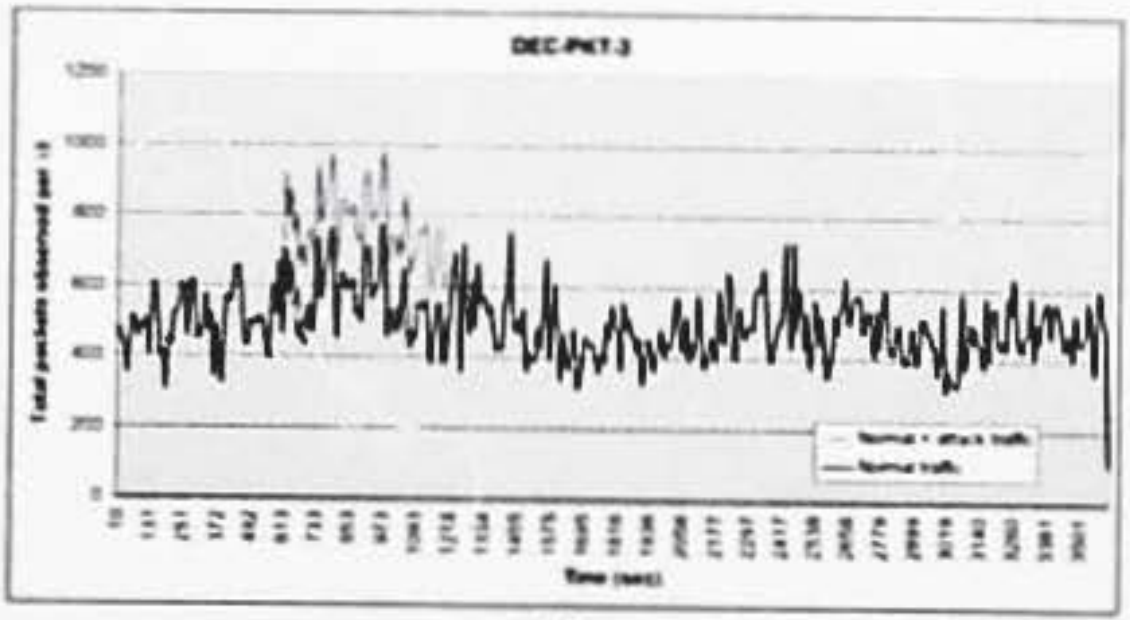

(c)

Figure 13: Traftic traces with and without attack traflic for DEC-PKT-1 (a), DEC. PKT-2 (b), and DEC-PKT-3 (c) 
Figure 13 ahove shows the difierence between the normal trafic traces and the traces with atrack traffic added. Note that the altach begins 10 minutes into the trace and lasts for 10 minutes.

Intereatingly, in the case of DEC-PKT-3, RSD detects $100 \%$ of a SYN flood when the rate is grealer than $20 \mathrm{SYN}$ packets per second. This is much higher compared to DEC. PKT-1, where RSD detected $100^{\circ}$. of a SYN flood when the rale was 6 SYN packets per second. This is due to the differing traflic conditions present in the two traces. A SYN flood is harder to detect when there is a greater amount of background traflic since it is easier for the atack packets to blend in with normal trafic. In this case, successful detection of a SYN flood requires a higher rate of attack packets compared to a low traflic scenario. Figure 14 confirms this observation by presenting a comparison between the traffic rales at the DEC site. It shous that the DEC-PKT-3 trace has much more traflic than the DEC-PKT-1 trace, which explains why RSD can pick up attacks with lower SYN flood rates in DEC.PKT-I.

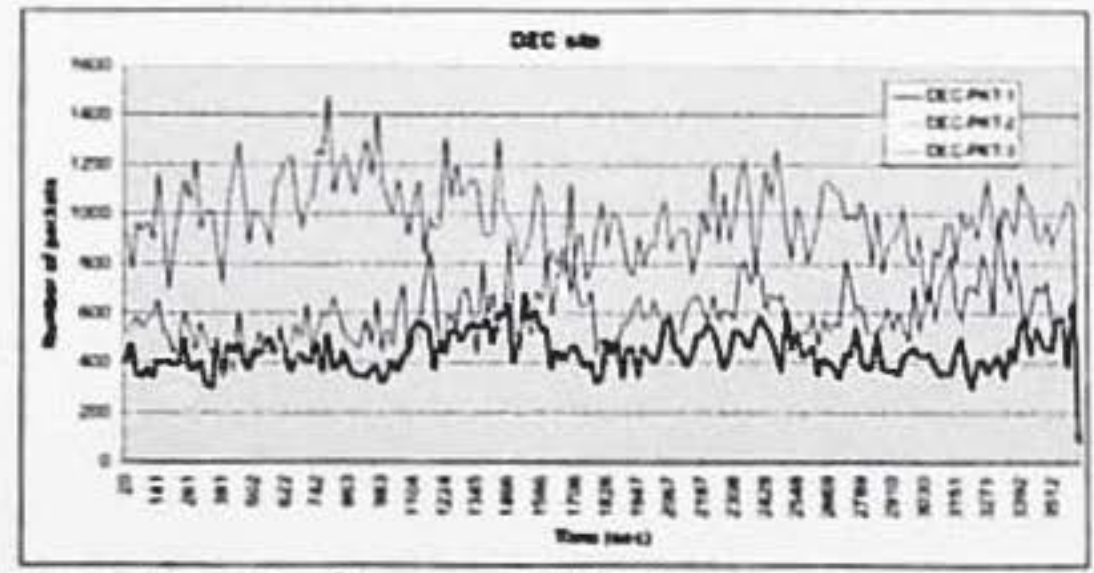

Figure 14: Comparison of trafile rates at DEC site 


\subsection{L.BL site results}

Results from simulated runs of RSD on the LBL traces are shown in Figure 15. From this figure, we can see that RSD can detect SYN flools with attack rates as low as I SYN pachet per second. As explained in the previous section, this is a result of a low traflic rate at the edge router. In this case, the LBL traces contain very little traflic, which results in easy detection of very low-rate SYN floods. Figure 16 shows a comparison of traffic rates at the LBL and DEC sites, which verifies the correlation between low irafic rates and low minimum SYN flood detection.

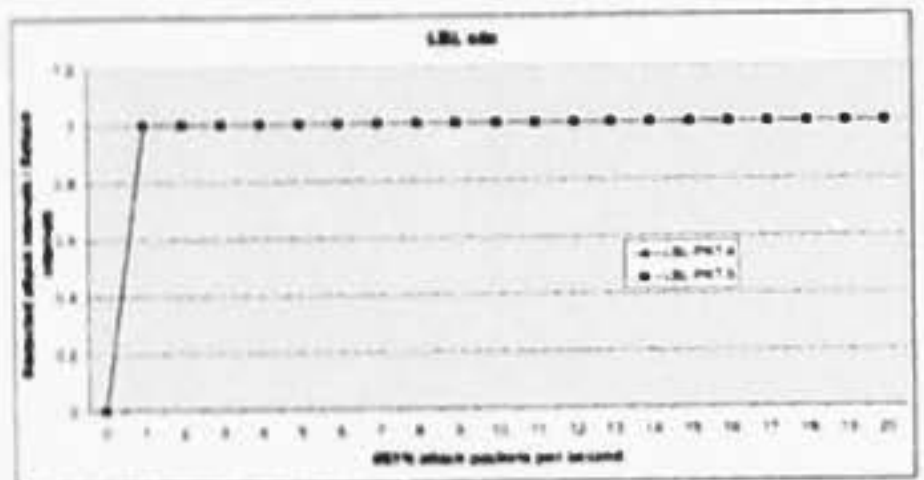

Figure 15: Resulis frnm RSD simulations (LBL slie)

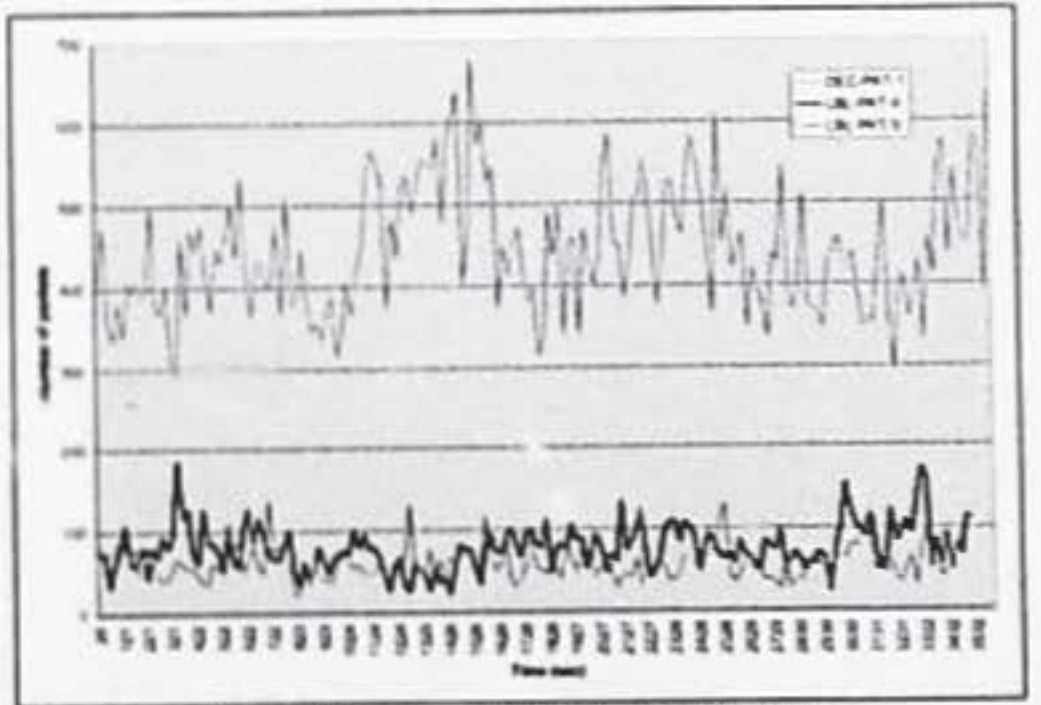

Figure 16: Comparion of traftic rales at DEC and LBL. sites 


\subsection{Discussion}

The previous sections lave shown how a typical implementation of RSD will perform using trace-driven simulations. Dala gathered from these simulations las shown the importance of optimizing At through the use of an ofline training plase. This enables RSD to quickly detect an attack without incurring a high false positive ratc.

The presence of varying traflic rates was also shown to have one of two effects on the performance of RSD: an increase in the FPR or a suboptimal (100 large) minimal delection time. These effects are mitigated in RSD by dynamically adapting At based on current trafic conditions. This ensures optimal performanse of the detection engine in the presence of long-term variations in trafic palterns.

Finally, the effectiveness of RSD was shown through the use of simulated attack traces, and performance data was gallered, based on the minimum SYN flood rate which results in a $100 \%$ attack detection rate. It was found that trafic rate, again, plays a key role in this metric. Lower background traffic mites make it easier to detect small mate SYN floods since the attack trafic is more anomalous. Concurrently, when larger background traflic rates are present, higher SYN flood rates are required to successfully detect an attack.

\section{4-3.1 SYN-dog Comparison}

Wang et. al. [42] have presented a similar solution to RSD called SYN-dog. Both take advantage of the correlation between SYN and SYN-ACK packets to detect DDOS 
aracks and both aim to detect anacks at the source networks. However, SYN-dog [42] uses a Cumulative Sum (CUSUM) method to detect SYN flood attacks rather than an adaptive threshold method, as in RSD. This results in a slightly higher degree of accuracy (less false positives) in SYN-dog, at the expense of a longer detection time. RSD, on the other hand, is more sensitive to anomalous traffic bursts and will throw more false positives compared to SYN-dog. This is an acceptable tradeoff for achieving our goal of fast detection time, which SYN-dog achieves.

SYN-dog does not feature a training phase to optimize $A t$ as in RSD. The authors of [42] simply set At to 20 seconds, thereby limiting the minimum detection time to this value. RSD. on the other hand, will determine an optimum $\Delta t$ during the training plase and will dynamically adjust the time inierval during execution. This allows RSD to keep the minimal attack detection time as small as possible, even smaller than SYN-dog in some cases. For example, in the case of the DEC-PKT-3 unfic mix, RSD features a minimum detection time of 10 seconds due to the training phase.

The ability of RSD to dynamically adjus $A t$ makes it more generally applizable to some network environments compared to SYN-dog, especially in low traffic situations. In these cases. SYN-dog's static interval of 20 seconds may not be enough time to obtain an adequate measurement of SYN-ACK packets, which ean result in a higher folse positive rate. For cxample, if only $10 \mathrm{SYN}$-ACK packets were seen in a single imterval, then a single missing SYN packet would seem particularly anomalous, possibly resulting in an attack condition. Most likely, this single missing packet would not indieate an aftack. 
Contrast this to the case where 100 SYN-ACK packets are observed and the some SYN packet is missing. In this case, an anomalous condition would not be observed. RSD aims for the latter case by dynamically adjusting $A t$ so the number of SYN-ACK packets observed per time interval is close to optimal. While this may result in a longer minimum detection time, it provides a greater degree of accuracy and makes RSD more effective over a wide range of network environments.

Similar to RSD, the efficacy of SYN-dog is examined in [42] using trace-driven simulations. The results are consistent with those found from simulations using RSDlarger flooding rates lead to faster and easier detection of attacks, and detection is much more sensitive in the presence of a lower volume of traffic.

42 


\section{CLAPTER 5: APPLICABILITY OF RSD TO NETWORK PROCESSORS}

As covered in section 2, network processors (NPs) luave been developed to provide highspeed packet processing with the flexibility of a programmable architecture. A key feature of RSD is its ability to dynamically adapt to changing traffic conditions. This requires on-the-fly calculations which will result in changes in the behavior of RSD. Additionally, RSD requires ofline training to optimize its variables, which then need to be programmed into the device. Clearly, network processors may provide RSD with the necessary platform for a successful and efficient implementation. NPs can give RSD the flexibility to modify its belavior while still maintaining line-speed packet processing. This section will explore the applicability of RSD on a network processor through the use of a NP simulator.

\subsection{Simulator}

The simulator used for performance testing of RSD is based on the SimpleScalar toolkit [50] and is designed to represent the arehitecture and performance of the Intel IXP 1200 network processor as shown in figure 17. The simulator gives a user the ability to simulate a generic out-of-order supersealar, taking the role of the StrongARMI processor and the 6 microengines, each of which contains 4 context-switchable threads for packet processing. Suppont for $\mathrm{C}++$ objects lus also been added to the simulator to increase its flexibility and ease of use. 


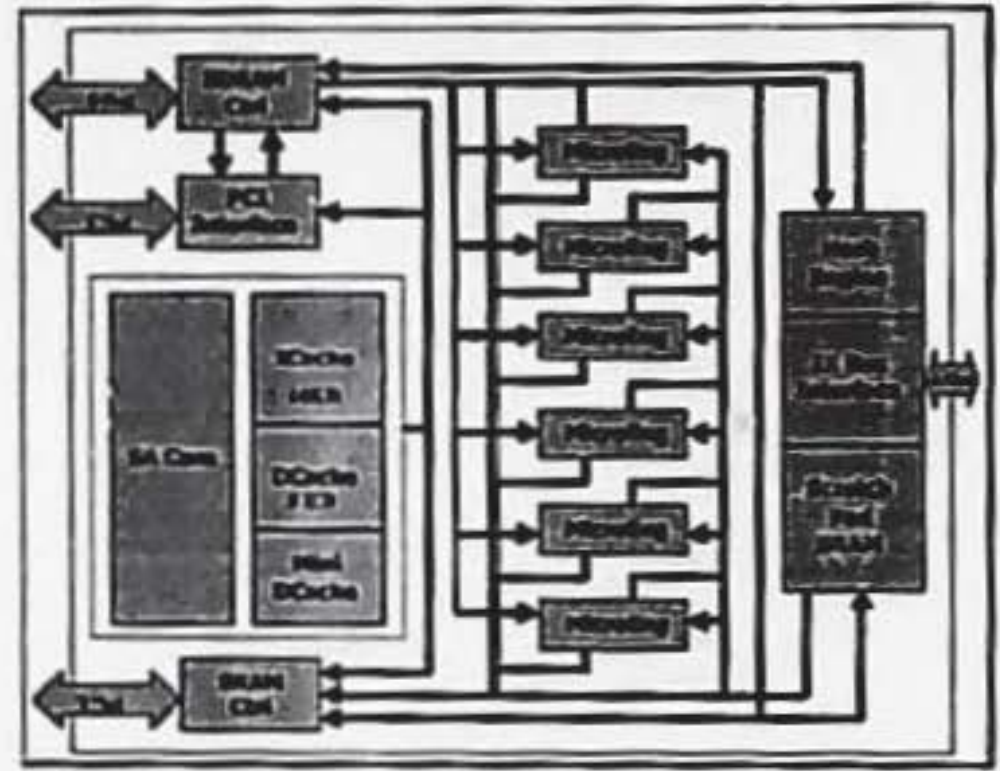

Figure 17: Intel IXP 1200 Architecture (from [49])

\section{Implementution Results}

The RSD algorithm was implemented in the simulator and simple performane: measurements were collected to investigate the basic feasibility of running RSD on a network processor.

For comparison purposes, a baseline implementation of simple router functionality was completed and cycle counts were collected using the DEC-PKT-1 trace. The baseline implementation simply parses the destination IP address field of each packet and stores it imto a temporary imemal variable. In a fully functioning router, a routing table lookup would be performed using this destination IP address and outgoing interface information would be obtained. but for the purposes of this investigation a reference cyele count is all that is needed. Results from the baseline implementation are shown in figure 18 (1). 


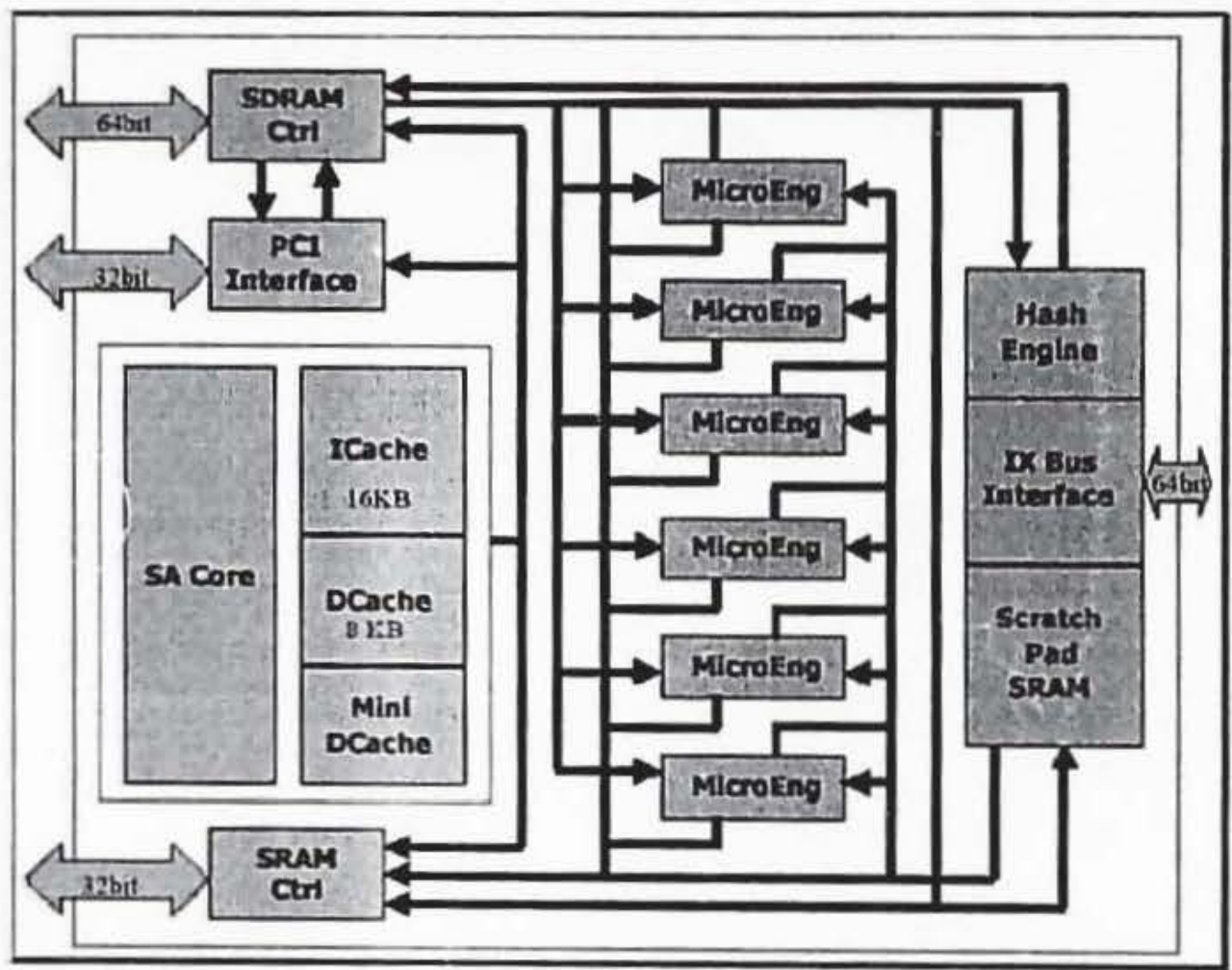

Figure 17: Intel IXP 1200 Architecture (from [49])

\subsection{Implementation Results}

The RSD algorithm was implemented in the simulator and simple performance 
The RSD algorithm was then implemented and cycle count data was collected during runs using the DEC-PKT-I trace. In the case of this trace, of line training was performed and $\Delta t$ was set to 10 seconds.

The RSD algorithm requires that each packet be inspeeted to determine if it is a TCP SYN or TCP SYN-ACK packet, which requires inspeetion of the TCP flags field in the TCP header. This functionality was added to the baseline implementation and the difference in cyele count was calculaled to determine the cast of the new functionality. Results showed that the cost of inspecting the TCP flags field and inerementing the appropriale counter ranged from II to 15 cyeles per packet, depending on the type of packet and the value in the TCP flag field. Results are shown in figure 18 (2). This represents an approximately 1 percent increase in total cycle count.

Next, the cost of calculating the ratio $R_{\infty}$ updating $D_{m}$ and checking for an attack condition was evaluated. These tasks are done every $4 t$ seconds, not for every packet. The total number of SYN-ACK packets $(E S A)$ is also compared to $S t_{\text {qet }}$ at this point, and At is adjusted as necessary to keep RSD running optimally. The cost for these calculations and updates ranged from 51 to 72 cyeles, depending on the behavior of RSD. Results are shown in figure 18 (3). This represents an additional 3-5 percent increase in total cycle count. 


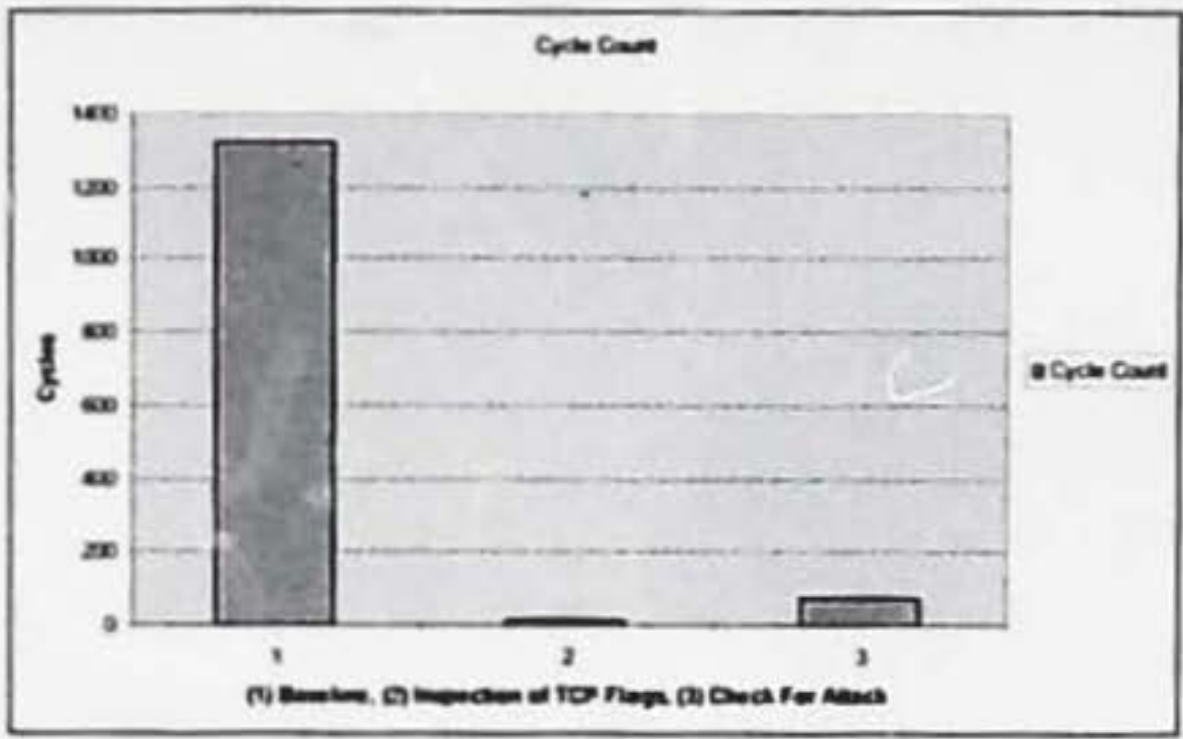

Figure 18: Results From Simulator. (I) represents total cycle count for the basellne implementation. (2) represents the additional cycles required for the inspection of the TCP nags field. (3) represents the additional cycles required w lien checking for an attack condition.

These results show that RSD has a minimal impact on the performance of a simulated network processor when performance is based solely on cyele counts. The addition of II to Is cycles per packet (a I pereent increase) should not present a problem for a network processor. and the additional 51 to 72 cyeles (a $3-5$ pereent increase) required when checking for an attack condition will only happen every $\boldsymbol{\Delta}$ seconds, not for every packet. In fact. Spalink et. al. [5] have shown that an additional 400 cycles are available in the LXP 1200 microengines for additional packet processing bejond simple IP forwarding. These results are encouraging and suggest that the processing requirements of RSD can easily be met by using a netuork processor. Additionally, these numbers were obtained using minimum-sized packets arriving at maximum speed on 8100 Mbps ports. When a more realistic traffic mix is introduced, such as the DEC-PKT-I trace, perfomance can be expected to inerease, resulting in more available cycles for additional packet processing 


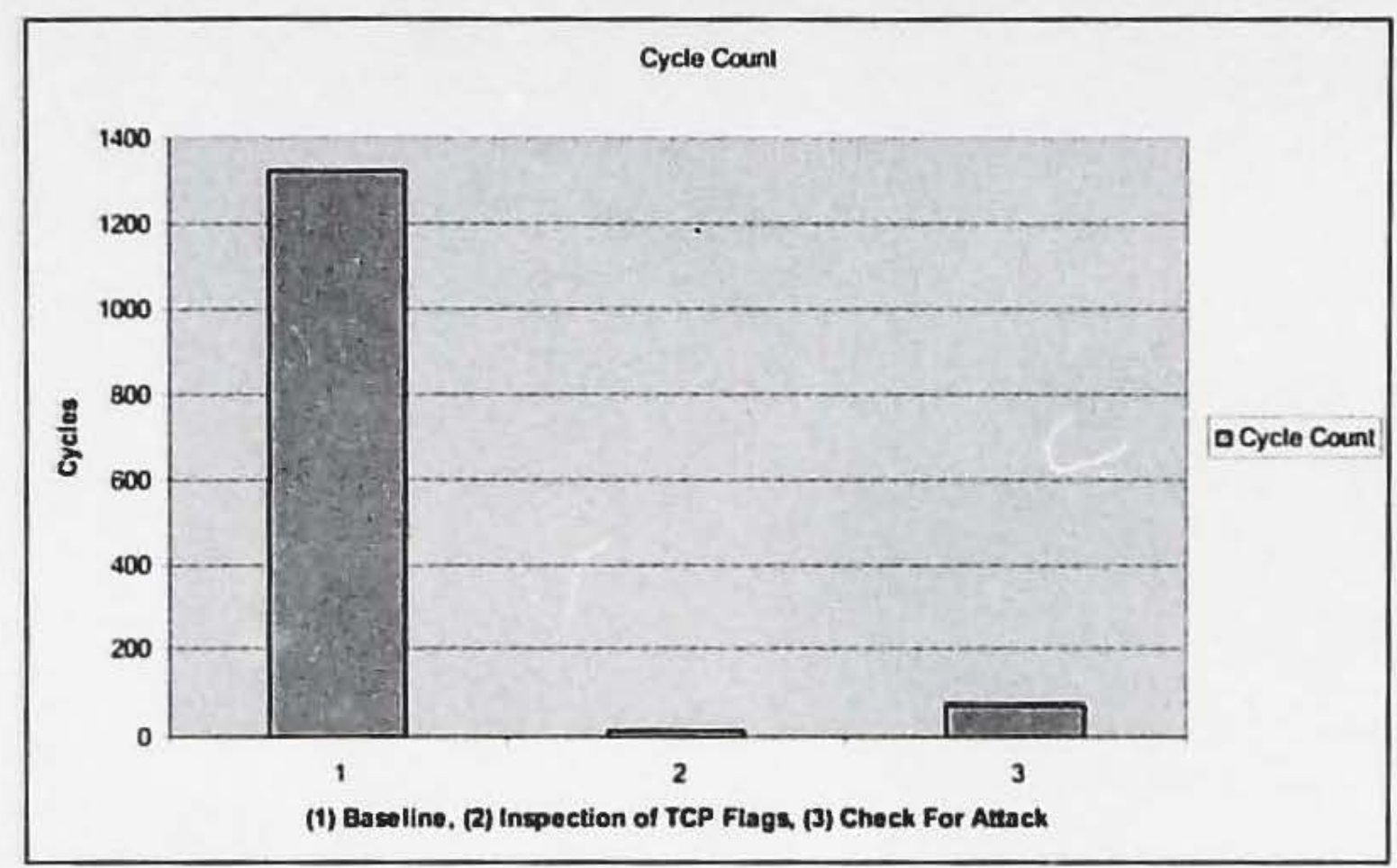

Figure 18: Results From Simulator. (1) represents total cycle count for the baseline implementation. (2) represents the additional cycles required for the inspection of the TCP flags field. (3) represents the additional cycles required when checking for an attack condition.

These results show that RSD has a minimal impact on the performance of a simulated network processor when performance is based solely on cycle counts. The addition of 11

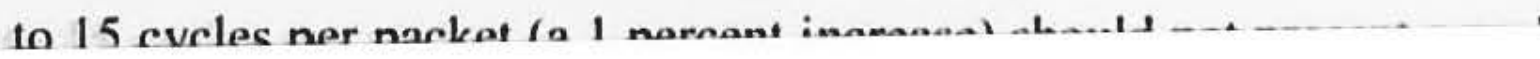




\section{CIIAITER 6: CONCLUSION}

This thesis presents a novel appronch to detecting a certain type of distributed denial-ofservice attack called a SYN flood. The detection algorithm, named Ratio-based SYN Flood Delection (RSD), is a type of adaptive threshold algorithm and features dynamically adjusting behavior to optimize its performanee depending on the networking environment. RSD is designed to detect SYN flood attacks close to their sources, and is therefore located on the link that connects a stub network to the Intemet. This allows attack trafic to be detected and filtered before it can enter the Internet and consume valuable bandwiditl.

An in-depth unalysis of RSD is presented. focusing on the tradeoffs between different values for parameters in the RSD algoritum. A methodology is discussed which can optimize these parameters to suit the particular traffic characieristics of the stub network.

The effieacy of this methodology is proven through the use of trace-driven simulations, and results are analyzed to determine the minimal mate of attack traffic which is needed for RSD to successfully detect $100 \%$ of the altack. For example, in the case of simulations using traces gathered from the Digital Equipment Corporation, RSD was able to detect an altack utilizing $8 \mathrm{SYN}$ packets per second in as little as 15 seconds. Traffic mete is shown to luave a signifieant effect on this performanee metric as well as the false positive ratio, demonstrating the need for the adaptive behavior present in RSD. 
Network processors may represent the ideal implementation environment for RSD, due to their flexibility and performance characteristies. RSD is implemented on a simple network processor simulanor and preliminary data is collected which shows minimal degradation of performance. This indicates that RSD can realistically be deployed on a network processor platform.

Detection of DDoS attacks will always be a difficult problem, especially in the face of determined attackers. The unpredictable and constanlly varying behavior of network traffic also makes it virtually impossible to detect attacks with $100 \%$ aceuracy. However. RSD is applieable to a wide range of deployments due to its optimizing methodology and adaptive behavior.

\subsection{Future Work}

Future work should further validate the performance of RSD on a wide range of network deployments, from lange institutional networks to small in-home networks. This may lead to further enhancements of the algorithm. Also, while the traces used in this thesis consisted of a shon time period, validation of the performance of RSD over long time periods should be explored. For example, long-term varintions in the traffic characteristics of a network may necessitate the need for another training phase al some point. Further study would need to be done to identify this point.

This thesis assumed that attacks were of a constant intensity and duration, which may or may not represent the current trend in nttack signatures. Attackers are always coming up with novel ways to defeat current detection schemes, so different styles of SYN flood 
macls should be simulated in orler to identify possible weaknesses in RSD. Improvements to RSD may be possible which could allow it to detect a wider range of anacl, incloding bursty and slow-growth attacks

RSD does not attermpt to identify the specific atuack packet flowx, it only detects when an atlack is present. Successful mitigation of a DDaS attack will require filtering capabilities to identify and drop the malicious packets. The design of a filtering algorithm is beyond the scope of this thesis, but the observation of the comelation between SYN and SYN-ACK packets may help in the design of such an algorithm. Statisties pathered by RSD could be of use to the filtering engine, resulting in a close cooperation between detection and filtering-

RSD was shown to be theoretically appropriate for implementation on a netuork processor, and a simple implementation was presented along with some basic results. Further work will need to be done to explore the tradeoffs and performance limitations associated with implenemting RSD on a network processor. This will require further development of the network processor simulator. Possible areas to explore include memory usage, tatency, and throughput. An in-lepth study into the tradeofis between the two different network processor arehitectures (serial and parallel) would also be extremely valuable. 


\section{REFERENCES}

[1] R. Hax, C. Jeffries, L Kencl, A. Kind, B. Metzler, R. Pletka, M. Waldvogel, L. Frelechoux, P. Droz, Creating Advanced Functions on Network Proceswen: Experience and Perspective", IEEE Netrouk, Vol. 17. No. 4, July 2003.

[2] P. Paulin, F. Karim, P. Bromley. "Network Processors: A Perspective on Market Requirements, Processor Architectures and Embedded S/W Tools". Proc. of DATE (Design, Automation, and Tess in Europe) 2001. March 2001.

[3] T. Spalink, S. Karlin, L Peternon, Y. Gottieb, -Building a Rolaus Softwarebased Rouler Using Network Processors". ACAI SIGOPS Operating Syztems Reviens. Proc. of the $18^{\text {th }}$ ACM Sympasiun an operuting systems principles. October 2001.

14] P. Crouley. J. Bact, "A Modeling Framework for Network Processor Systems", HPCA-8 Worklhop an Nefwork Processors, Cambridge, MA, Februrary 2002.

[5] T. Spalink, S. Karlin, L Peterson, Evaluating Network Processors in IP Forwarding-. Princeton University Teclunical Report TR-626-00, January 2001.

|6| J. Allen, B. Bass, C. Basso, R. Boivic, J. Calvignac, G. Davis, L. Frelechoux, M. Heddes, A. Herkendorf, A. Kind, J. Logan, M. Peyravian, M. Rinaldi, R. Sabhiki, M. Siegel. M. Waldvogel. -PowerNP Network Processor. Hardware. Software, and Applications". IRM Journal of Research and Development, Vol. 47. No. 2/3. Mlarch/May 2003.

[7] Y. Lin, Y. Lin, S. Yang. Y. Lin, "Diffserv over Network Proceswors: Implemeniation and Evaluation". Proc. of IEEE $/ \sigma^{4}$ Symposium on /lot Interconnects, Stanford University. Palo Alto. Augus: 2002.

[8] M. Burns, G. Prier. J. Airkovic, P. Reiher, -Implementing Address Assurance on the Intel LXP Router". Proc. of NPC 2002, 2002.

[9] T. Aoki, -Implementation Methods of Differentiated Services by Using CBQ with Dynamic Bandwidth Decision Meltod for Network Precessors". Master's Thesis, University of Tsukubs, 2002.

I10; Intel LXA University Program Roster, htip:/wwww.ixacdu.condroster/, 2003.

[II] R. Chang, -Defending against Fooding-Based Distrituted Denial-of-Service Attacks: A Tutorial", IEEE Conmunications. Vol. 40, No. 10, October 2002.

[12] T. Peng. C. Leckic, K. Ramamohanaroa, "Detecting Distributed Denial of Service Attacks Using Sourxe IP Aduress Monitoring". Unibersity of Afelbourne Tectuical Report. November 2002. 
[13] T. Peng, C. Leckic, K. Ramamohanaroa, "Protection from Distribuled Denial of Service Attack Using History-based IP Filtering". JEEE Int. Conf. on Canmunications (ICC) 2003, Anchorage, Alaska, May 2003.

[14] T. M. Gil, M. Poletter, "MULTOPS: A dala-sinucture for bandwidth altack detection", In Proceedings of USENIX Security Symprasium 200I, Washinglon, D.C., August 2001.

[15] K. Park, II. Lee, "On the effectiveness of rouic-based packet filtering for Distributed DoS altack prevention in power-law intemets", In Proceedings of ACM SIGCOMA 2001. San Diego, CA, August 2001.

[16] H. Wang, D. Zlung, K.G. Shin, "Detecting SYN Flooding Altacks", In Proceedings of IEEE INFOCOAI 2002, New York City, NY, June 2002.

[17] P. Ferguson, D. Senie, "Network Ingress Filtering: Defeating Denial of Service Attacks Which Employ IP Source Address Spoofing", RFC 2827, May 2000.

[18] J. Mirkovic, G. Prier, P. Reiher, "Attacking DDoS at the Source". In Procedings of the ICNP. Paris, France, November 2002.

|19] J. Li, J. Mirkovic, M. Wang. P Reiher, L. Zhang. "SAVE: Source Address Validity Enforcement Prolocol", In Proceedings of INFOCOM 2002, New York City, NY, June 2002.

[20] J. Mirkovic, J. Mantin, P. Reiher, "A Taxonomy of DDaS Altacks and Defense Mectranisms", UCLA CSD Technical Repon CS-TR-020018.

[21] J. Mirkovic, G. Prier, P. Reiher, "Souree-End DDoS Defense", In Procredings of Nenwork Computing and Applications (NCA) 2003. Cambridge, MLA, April 2003.

[22] J. Mirkovic, Z Xu, J. Li, M. Sclinaider, P. Reiher, L Zhang, "iSAVE: Inerementally Deployable Source Address Validation", UCLA CSD Teclinical Report CS-TR-020030.

[23] C. Jin, II. Wang. K. Shin, "Ilop-Count Filtering: An Effective Defense Against Spoofed Traffic", In Proceedings of $10^{6}$ ACM Computer and Communication Security, Washingion, DC, 2003.

[24] A. Hussain, J. Ileidemann, C. Papadopoulos, "A Framework for Classifying Denial of Service Auncks", In Proceredings of 2003 Corf. on Applications. Technologies, Anchitectures, and Protocols for Computer Conmumictaions, Karlsnutic, Germany, 2003. 
[25] J. Xu, "Sustaining Availability of Web Services under Distriluted Denial of Service Altacks", IEEEE Transactions on Compusers, Vol. 52, No. 2, February 2003.

[26] CERT Coordination Center, "Denial of Service Altacks", http://suww.cert.org/iech_sipydlenlal_of_senice.html.

[27| CERT Advisory CA-1997-28, "IP Denial-of-Service Altacks", Hut://www.cert.org/advisories/CA-1997-28.html.

|28| ClERT Advisory CA-1996-26, "Denial-of-Service Allack via ping",

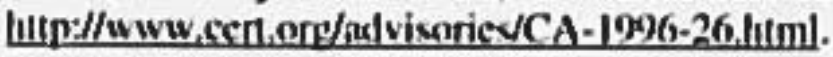

[29] Distributed Denial of Service (DDoS) Allack viools. щu://saff.wrishington.ceduddiuricl/misc/ddoy, January 2004.

[30] V. Paxon, “An Analysis of Using Reflectors for Distributed Denial-Or-Service Auncks", ACM Computer Communications Review (CCR), Vol. 31, No. 3, July 2001.

[3I] CERT Incident Note IN-2000.04, "Denial of Service Attacks Using

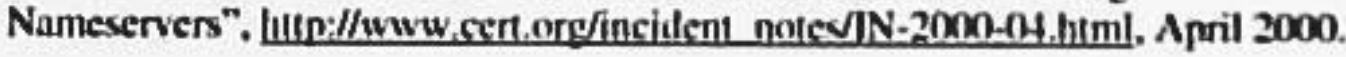

|32| S. Gibson, "Distributed Reflection Denial of Service: Description and analysis of a potent, increasingly prevalent, and worrisome Intemet altack". Lilin://grc.comilovilnilox.lim, February 2002.

[33] Mazu Networks, hu1p://www.mazunctworks.com.

[3-1] SonicWall, hulp://wwwsonicwall.com

[35] FortiNet, ltup://www.fontinet.com

[36] Cisco Systems, hu1p://www.cisco.com

[37| D.E. Drodsky, B.S. Darkhovsky. Nonnanmetric Melhos/s in Chanscancin Prolulems, Kluwer Acudemic Publishers, 1993.

[38] R.B. Dlazek, II. Kim, B. Rozovskii, A. Tartakovaky, "A Novel Approach to Detection of Denial-of-Service Attacks via Adaptive Sequential and Batchsequential Clange-point Detection Mlethods", In Procerdings of IEEE Systems, San and Cybernetics Information Assumance Workshop, June 2001.

[39] D. Yau, J. L.ui, IF. Liang, "Defending Against Distributed Denial-of-senvice Atuaks with Mlax-min Fair Server-centric Router Throtlles", In Pramedings of 
IEEE International Wiorkshop on Quality of Senice (IWgaS), Mliami Beach. FL. Nlay 2002

140| 1. Jung B. Kristinamunhy, M. Rahinovich, "Flash Crowds and Denial of Service Attacks Characterization and Implications for CDNs and Web Site", In Procentings of $1 I^{*}$ Warld Wide Web Conference, Ilonolulu, HI, May 2002.

141] A. Belenky, N. Ansuri, On IP Tracebach", IEEE Communicutions Magazine, Vol. 41. No. 7. July 2003 .

|42] H. Wang, D. Zhang, K. Shin, SYN-dog: Sniffing SYN Flooding Sources", In Proccendings of IEEE ICDCS 2002. Vienna, Austria, 2002.

[43] G. Malan et. al_-Otwervations and Experiences Tracking Denial-Of-Service Anacks Across a Large Regional tSP. Teclunical Report, Arbor Networks, 2001.

[4] D. Moore, G. Voeller, S. Savage, "Inferring Imernet Denial of Service Activity". In Preccedings of USENDX Seczurity Simpeosium 2001. Augusi 2001.

[45] W. Steven ICP/P Illusiraled, Volume 1. Addision-Wesley Publishing Company, 199.

[46] M. Basseville. I. Nikiforov, Deicction of Ahrup Chanecs: Theory and deplication. Prentice Hall, 1993.

147] B. Brodsky, B. Darhhowshy. Non-parametric Methods in Chanes-point Prohlems, Kluwer Academic Publishers, 1993.

148] The Imernet Traffic Archive". hrif-/fita ce.thl, pox, Lawrence Berkeley National Laboratory.

[49] E. Johnson, A. Kunze. IXP 1200 Programming, Intel Press 2002.

[S0] D. Burger. T. Austin. The SimpleSealar Tool Set. Version 20". hurf/lwuw. simplevealar.com, 2001. 